CRYSTALLOGRAPHIC COMMUNICATIONS

ISSN 2056-9890

Received 26 February 2018

Accepted 5 March 2018

Edited by $\mathrm{H}$. Stoeckli-Evans, University of Neuchâtel, Switzerland

Keywords: crystal structure; ruthenium(II); $\eta^{6}$ arene complex; phosphine complex; reductive amination; carbon monoxide; synchrotron radiation; hydrogen bonding; $\mathrm{C}-\mathrm{H} \cdots \pi$ interactions.

CCDC reference: 1827568

Supporting information: this article has supporting information at journals.iucr.org/e

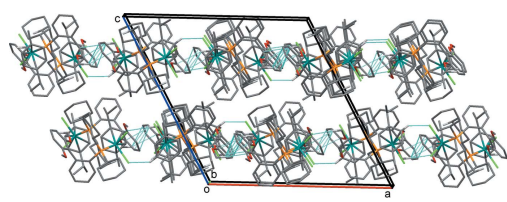

OPEN $\odot$ ACCESS

\section{Synthesis, crystal structure and catalytic activity in reductive amination of dichlorido $\left(\eta^{6}-p\right.$-cymene $)\left(2^{\prime}-\right.$ dicyclohexylphosphanyl-2,6-dimethoxybiphenyl- $\kappa P)$ ruthenium(II)}

\author{
Maria Makarova, ${ }^{a}$ Alexey A. Tsygankov, ${ }^{\text {a } *}$ Olga Chusova, ${ }^{\text {b Ivan V. Linko, }}{ }^{\mathrm{c}}$ Pavel V. \\ Dorovatovskii $^{\mathrm{d}}$ and Yan V. Zubavichus ${ }^{\mathrm{d}}$
}

\begin{abstract}
${ }^{a}$ Mendeleev University of Chemical Technology of Russia, Miusskaya sq. 9, Moscow 125047, Russian Federation, ${ }^{\mathbf{b}}$ Research Institute of Chemistry, Peoples' Friendship University of Russia (RUDN University), 6 Miklukho-Maklay St., Moscow 117198, Russian Federation, 'Inorganic Chemistry Department, Peoples' Friendship University of Russia (RUDN University), 6 Miklukho-Maklay St., Moscow 117198, Russian Federation, and ${ }^{\mathbf{d}}$ National Research Centre Kurchatov Institute, 1 Acad. Kurchatov Sq., Moscow 123182, Russian Federation. *Correspondence e-mail: alexey.a.tsygankov@gmail.com
\end{abstract}

The title compound, $\left[\mathrm{RuCl}_{2}\left(\mathrm{C}_{10} \mathrm{H}_{14}\right)\left(\mathrm{C}_{26} \mathrm{H}_{35} \mathrm{O}_{2} \mathrm{P}\right)\right]$ (I), crystallizes in the monoclinic space group $P 2_{1} / c$ with two crystallographically independent molecules $(A$ and $B)$ in the asymmetric unit. The geometries of both molecules are very similar and distinguished only by the twist angles of the two benzene rings in the phosphine substituents [89.54 (14) and $78.36(14)^{\circ}$ for molecules $A$ and $B$, respectively]. The $\mathrm{Ru}$ atoms have classical pseudo-tetrahedral pianostool coordination environments. The conformation of each molecule is stabilized by intramolecular $\mathrm{C}-\mathrm{H} \cdots \mathrm{O}$ and $\mathrm{C}-\mathrm{H} \cdots \mathrm{Cl}$ hydrogen bonds and $\mathrm{C}-\mathrm{H} \cdots \pi$ interactions. The two molecules are linked by a $\mathrm{C}-\mathrm{H} \cdots \mathrm{Cl}$ hydrogen bond. In the crystal, the molecules are further linked by $\mathrm{C}-\mathrm{H} \cdots \pi$ interactions, forming $-A-B-A-B-$ chains propagating along the $a$-axis direction. Complex $\mathbf{I}$ is an active catalyst for reductive amination reaction. The catalytic activity of this complex can be explained by the lability of the $p$-cymene ligand, which can be replaced by two-electron ligands such as $\mathrm{CO}$ or amine.

\section{Chemical context}

The design of new organometallic complexes is important for the development of new catalytic processes as well as for understanding those already known. Recently, a new methodology for reductive amination in the presence of carbon monoxide as the reducing agent, catalysed by rhodium (Chusov \& List, 2014; Afanasyev et al., 2016; Yagafarov et al., 2015), iridium (Moskovets et al., 2017; Molotkov et al., 2017) and ruthenium (Kolesnikov et al., 2015; Afanasyev et al., 2017) has been described. This protocol is based on the deoxygenation potential of $\mathrm{CO}$ and does not require an external hydrogen source. This methodology is therefore potentially more selective for those substrates bearing groups that are sensitive to hydrogenation. As a result of the high cost of rhodium and iridium, the development of new catalytic systems based on more abundant metals is important. It has previously been shown that addition of phosphines to ruthenium systems, which were supposed to stabilize catalytic species, dramatically decreases the activity of the catalytic system. To further understand this process and the role of phosphines, the title complex, I, was synthesized and its crystal structure and catalytic properties are reported herein. 


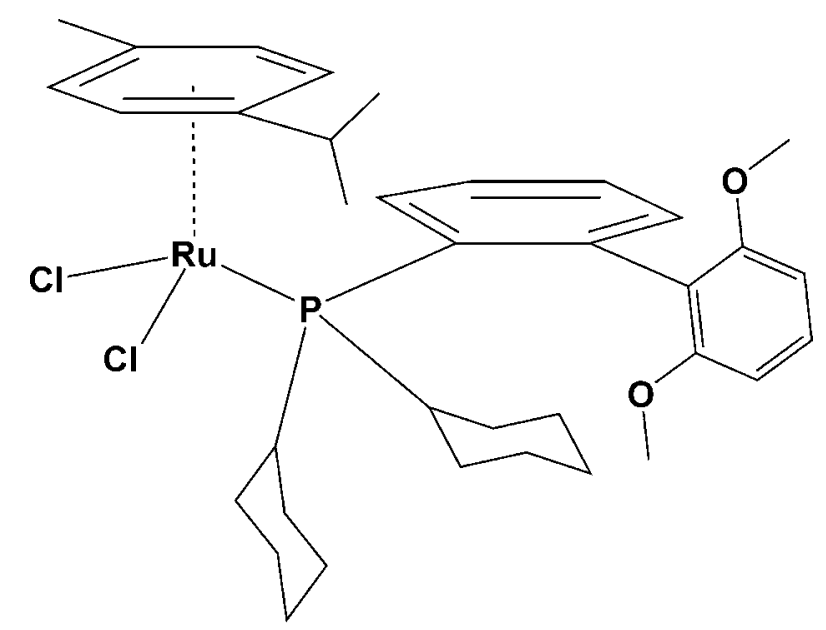

Such $\eta^{6}$-arene $\mathrm{Ru}^{\mathrm{II}}$ complexes with piano-stool coordination are known to be active catalysts in different processes (Therrien, 2009), including hydrogenation (Moldes et al., 1998), hydroboration (Kaithal et al., 2016), transfer hydrogenation (Aznar et al., 2013; Cerón-Camacho et al., 2006; Clavero et al., 2016) and isomerization of allylic alcohols (Díaz-Álvarez et al., 2006; Baraut et al., 2015). Moreover, such complexes have shown promising medicinal properties (Nazarov et al., 2014), including anticancer activity (Chuklin et al., 2017).

\section{Structural commentary}

The title compound, I, crystallizes in the monoclinic space group $P 2_{1} / c$ with two crystallographically independent molecules ( $A$ and $B$, comprising Ru1 and Ru2, respectively) in the asymmetric unit (Fig. 1). The geometries of both molecules are very similar, as illustrated in Fig. 2, showing the molecular overlap of the inverted molecule $B$ on molecule $A$ [r.m.s. deviation of $0.227 \AA$ А Mercury (Macrae et al., 2008)]. They are distinguished only by the twist angles of the two benzene rings

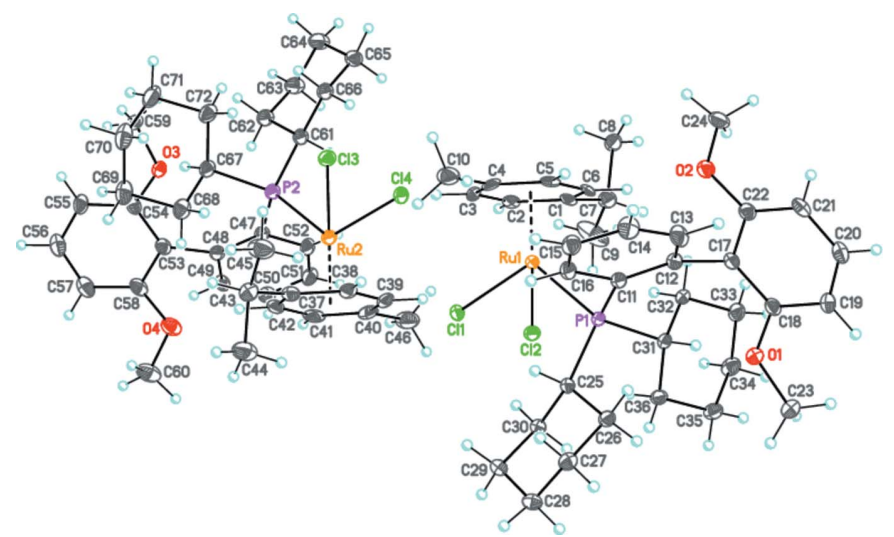

Figure 1

A view of the molecular structure of compound $\mathbf{I}$, with atom labelling. Displacement ellipsoids are shown at the $50 \%$ probability level.
Table 1

Hydrogen-bond geometry $\left(\AA,^{\circ}\right)$.

$\mathrm{Cg} 3$ and $\mathrm{Cg} 8$ are the centroids of rings $\mathrm{C} 17-\mathrm{C} 22$ and $\mathrm{C} 53-\mathrm{C} 58$, respectively.

\begin{tabular}{lllll}
\hline$D-\mathrm{H} \cdots A$ & $D-\mathrm{H}$ & $\mathrm{H} \cdots A$ & $D \cdots A$ & $D-\mathrm{H} \cdots A$ \\
\hline $\mathrm{C} 6-\mathrm{H} 6 \cdots \mathrm{O} 2$ & 0.95 & 2.50 & $3.328(6)$ & 146 \\
$\mathrm{C} 30-\mathrm{H} 30 A \cdots \mathrm{Cl} 2$ & 0.99 & 2.74 & $3.552(4)$ & 139 \\
$\mathrm{C} 45-\mathrm{H} 45 C \cdots \mathrm{Cl} 3$ & 0.98 & 2.79 & $3.577(5)$ & 137 \\
$\mathrm{C} 46-\mathrm{H} 46 C \cdots \mathrm{Cl} 4$ & 0.98 & 2.68 & $3.302(4)$ & 122 \\
$\mathrm{C} 62-\mathrm{H} 62 A \cdots \mathrm{O} 3$ & 0.99 & 2.58 & $3.261(6)$ & 126 \\
$\mathrm{C} 66-\mathrm{H} 66 B \cdots \mathrm{Cl} 4$ & 0.99 & 2.71 & $3.388(4)$ & 126 \\
$\mathrm{C} 72-\mathrm{H} 72 A \cdots \mathrm{Cl} 3$ & 0.99 & 2.78 & $3.617(6)$ & 143 \\
$\mathrm{C} 38-\mathrm{H} 38 \cdots \mathrm{Cl} 1$ & 0.95 & 2.70 & $3.376(4)$ & 129 \\
$\mathrm{C} 33-\mathrm{H} 33 B \cdots C g 3$ & 0.99 & 2.97 & $3.703(5)$ & 132 \\
$\mathrm{C} 69-\mathrm{H} 69 A \cdots C g 8$ & 0.99 & 2.91 & $3.649(6)$ & 132 \\
$\mathrm{C} 60-\mathrm{H} 60 B \cdots C g 3^{\mathrm{i}}$ & 0.98 & 2.84 & $3.655(6)$ & 142 \\
$\mathrm{C} 24-\mathrm{H} 24 B \cdots C g 8^{\mathrm{ii}}$ & 0.98 & 2.85 & $3.710(6)$ & 147
\end{tabular}

Symmetry codes: (i) $x-1, y, z$; (ii) $x+1, y, z$.

in the phosphine substituents $\left[89.54(14)^{\circ}\right.$ for $\mathrm{A}$ and $78.36(14)^{\circ}$ for $\left.B\right]$.

The ruthenium atom in each molecule has a classical pseudo-tetrahedral piano-stool coordination environment, being ligated by two chlorides, the phosphine 2-dicyclohexylphosphino-2,6'-dimethoxybiphenyl (SPhos) and an $\eta^{6}-p$-cymene ligand. Owing to steric hinderance, the average $\mathrm{Ru}-\mathrm{C}[2.248(3) \AA], \mathrm{Ru}-\mathrm{P}$ [2.4194 (11) $\mathrm{A}]$ and $\mathrm{Ru}-\mathrm{Cl}$ [2.4455 (11) $\AA$ ] bond lengths are slightly elongated in comparison with those observed previously in related ruthenium complexes (Muller \& Davis, 2012; Granville et al., 2012). The bond angles $\mathrm{Cl}-\mathrm{Ru}-\mathrm{Cl}\left[88.04(4)^{\circ}\right.$ for $A$ and $86.26(4)^{\circ}$ for $B]$ and $\mathrm{Cl}-\mathrm{Ru}-\mathrm{P}[87.23$ (4) and 87.53 (4) for $A$ and $87.86(4), 87.28(4)^{\circ}$ for $B$ ] fall within the normal range for known analogous complexes. The methoxy groups are coplanar to the parent benzene rings (r.m.s. deviations are $0.070 \AA$ for $A$ and $0.082 \AA$ for $B$ ). In each molecule there are

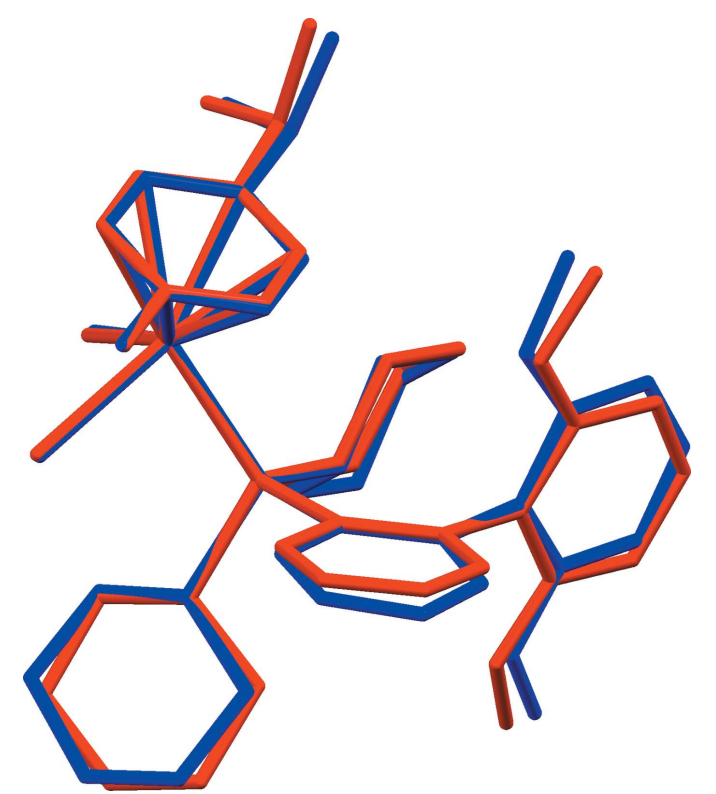

Figure 2

A view of the molecular overlap of the inverted molecule $B$ (red) on molecule $A$ (blue). H atoms have been omitted for clarity. 


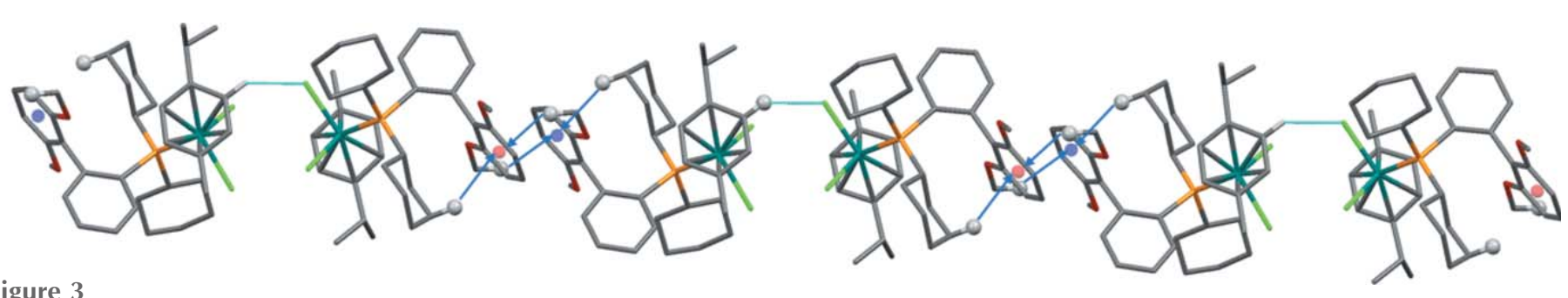

Figure 3

A view of the $\mathrm{C}-\mathrm{H} \cdots \mathrm{Cl}$ hydrogen bonds (dashed lines) and the $\mathrm{C}-\mathrm{H} \cdots \pi$ interactions (blue arrows) leading to the formation of chains along [100]; see Table 1 for details. Only the $\mathrm{H}$ atoms involved in these interactions are shown, and the centroid in molecule $A$ is red, while the centroid in molecule $B$ is blue.

intramolecular $\mathrm{C}-\mathrm{H} \cdots \mathrm{O}$ and $\mathrm{C}-\mathrm{H} \cdots \mathrm{Cl}$ hydrogen bonds and $\mathrm{C}-\mathrm{H} \cdots \pi$ contacts present (see Table 1), and the two molecules are linked by the C38-H38 . Cl1 hydrogen bond (Table 1 and Fig. 3).

\section{Supramolecular features}

In the crystal of $\mathbf{I}$, molecules are linked by a $\mathrm{C}-\mathrm{H} \cdots \mathrm{Cl}$ hydrogen bond and $\mathrm{C}-\mathrm{H} \cdots \pi$ interactions forming $-A-B-A-$ $B$ - chains propagating along [100]; details are shown in Fig. 3 and Table 1. The overall packing in the crystal structure of $\mathbf{I}$ is illustrated in Fig. 4. There are no other significant intermolecular interactions present in the crystal structure.

\section{Catalytic activity}

The catalytic activity was investigated in a model reductive amination reaction between $p$-tolualdehyde and $p$-anisidine in conditions similar to those reported previously for ruthenium systems (Fig. 5). We were delighted to find out that complex I was active and furnished the desired amine in $61 \%$ yield. The catalytic activity of this complex can be explained by the lability of the $p$-cymene ligand, which can be replaced by twoelectron ligands such as $\mathrm{CO}$ or amine. The role of the phos- phine ligand is in the stabilization of catalytically active species $\left[\mathrm{RuCl}_{2} \mathrm{SPhos} L_{x}\right]$. Interestingly, the dimeric precursor of $\mathbf{I}-[\mathrm{Ru}(p \text {-cymene }) \mathrm{Cl}]_{2} \mathrm{Cl}_{2}$ - was two times less active (the amine yield is $34 \%$ ), which can be explained by dissociation of the $p$-cymene ligands followed by aggregation of non-stabilized $\mathrm{RhCl}$ species. In summary, complex $\mathbf{I}$ is an active catalyst for reductive amination, and further tuning of phosphine ligands may result in even more active complexes.

\section{Procedure for reductive amination}

A glass vial in a $10 \mathrm{ml}$ stainless steel autoclave was charged with $0.5 \mathrm{~mol} \%$ of the catalyst, $\mathrm{CH}_{3} \mathrm{CN}, 1.2$ equiv. of the $p$-anisidine and 1 equiv. of the $p$-tolualdehyde (the use of a glass vial is crucial: interaction of the catalyst with the metal surface inside the autoclave can lead to decreased catalytic activity). The autoclave was sealed, flushed three times with 5 bar of carbon monoxide $(\mathrm{CO})$, and then charged with 50 bar of $\mathrm{CO}$. The reactor was placed in an oil bath preheated to $413 \mathrm{~K}$. After the indicated time, the reactor was cooled to room temperature and depressurized. The residue was purified by flash chromatography on silica gel using dichloromethane as eluent. ${ }^{1} \mathrm{H}$ NMR $\left[400 \mathrm{MHz}, \mathrm{CDCl}_{3}, \delta(\mathrm{ppm})\right.$, $J(\mathrm{~Hz})]: 7.27(d, J=8.1,2 \mathrm{H}), 7.16(d, J=8.1,2 \mathrm{H}), 6.79(d, J=$

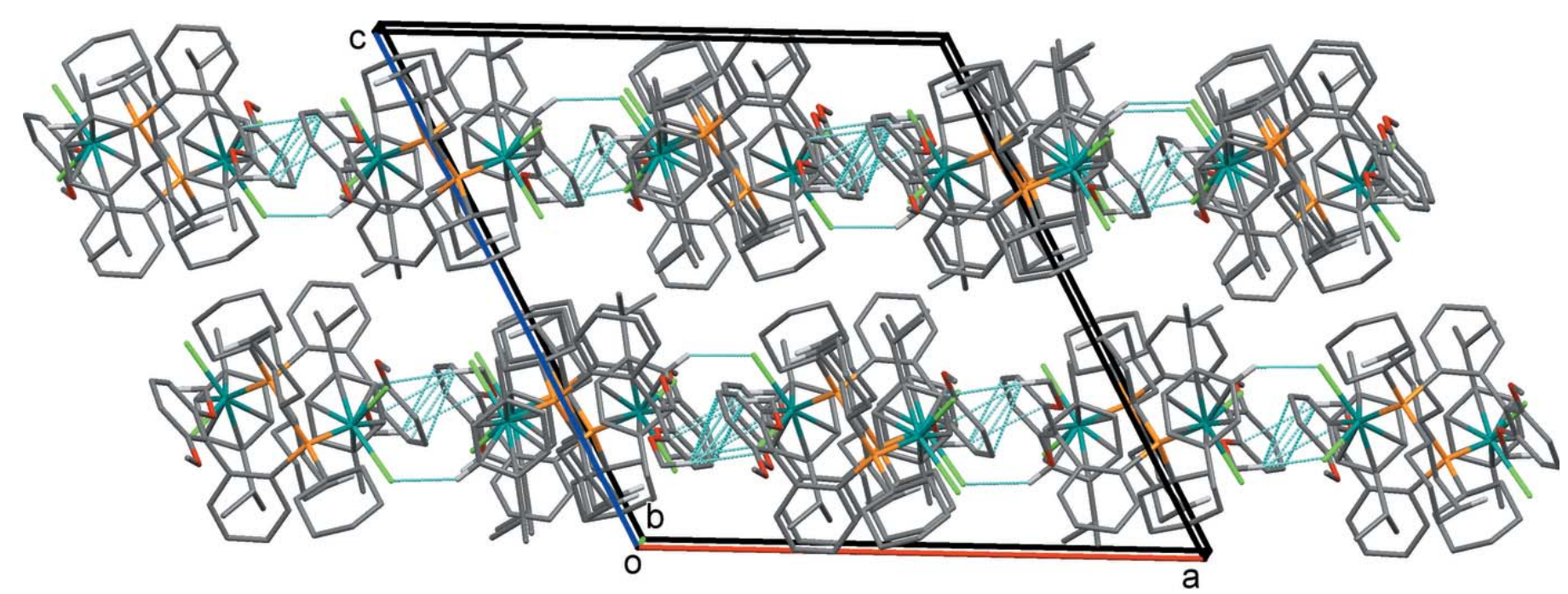

Figure 4

A view along the $b$ axis of the crystal packing of compound $\mathbf{I}$. The intermolecular interactions are shown as dashed lines (see Table 1), and only those $\mathrm{H}$ atoms involved in these interactions have been included. 


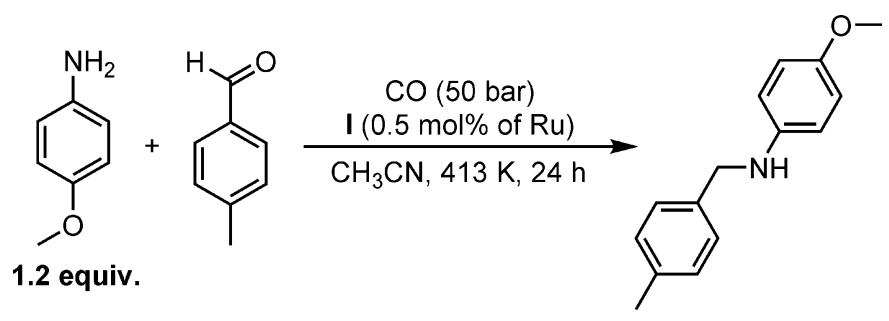

Figure 5

A model reductive amination reaction between $p$-tolualdehyde and $p$ anisidine catalyzed by complex $\mathbf{I}$.

$8.8,2 \mathrm{H}), 6.61(d, J=8.8,2 \mathrm{H}), 4.25(s, 2 \mathrm{H}), 3.75(s, 3 \mathrm{H}), 2.36(s$, $3 \mathrm{H})$.

\section{Synthesis and crystallization}

To a dichloromethane $(7 \mathrm{ml})$ solution of $\left[(p \text {-cymene }) \mathrm{RuCl}_{2}\right]_{2}$ $(0.050 \mathrm{~g}, 0.082 \mathrm{mmol})$ was added SPhos $(69 \mathrm{mg}, 0.168 \mathrm{mmol})$. The dark-orange solution was stirred at room temperature for $24 \mathrm{~h}$. The mixture was partially evaporated under reduced pressure, and the complex precipitated with diethyl ether $(10 \mathrm{ml})$ to give a dark-orange solid (37 mg, 66\%). Darkorange prismatic crystals were obtained by slow diffusion of pentane into the dichloromethane solution of complex $\mathbf{I}$.

Spectroscopic data: ${ }^{1} \mathrm{H}$ NMR $\left[\mathrm{CDCl}_{3}, 600 \mathrm{MHz}, 230 \mathrm{~K}, \delta\right.$ $(\mathrm{ppm}), J(\mathrm{~Hz})]: 8.24(d d, J=13.1,7.7,1 \mathrm{H}), 7.43-7.31(m, 3 \mathrm{H})$, $6.86(d, J=7.2,1 \mathrm{H}), 6.71(d d, J=23.2,7.2,2 \mathrm{H}), 5.46(d, J=6.2$, $1 \mathrm{H}), 5.32(d, J=6.2,1 \mathrm{H}), 5.16(d, J=6.1,1 \mathrm{H}), 5.05(d, J=5.8$, $1 \mathrm{H}), 3.82(\mathrm{~s}, 3 \mathrm{H}), 3.73(\mathrm{~s}, 3 \mathrm{H}), 2.72-2.52(\mathrm{~m}, 3 \mathrm{H}), 2.40(q, J=$ $12.9,1 \mathrm{H}), 1.93(d, J=12.1,1 \mathrm{H}), 1.73(q, J=12.6,1 \mathrm{H}), 1.61(s$, $4 \mathrm{H}), 1.65-1.43(m, 6 \mathrm{H}), 1.39-1.26(m, 4 \mathrm{H}), 1.18(d d, J=17.5$, $6.8,6 \mathrm{H}), 1.26-1.14(m, 1 \mathrm{H}), 1.08-0.92(m, 3 \mathrm{H}), 0.42(q, J=13.2$, $1 \mathrm{H}), 0.10(q, J=13.2,1 \mathrm{H}) .{ }^{13} \mathrm{C} \mathrm{NMR}\left[\mathrm{CDCl}_{3}, 151 \mathrm{MHz}, 230 \mathrm{~K}\right.$, $\delta(\mathrm{ppm}), J(\mathrm{~Hz})]: 158.3,157.5,139.6(d, J=27.6), 136.3,135.9$ $(d, J=15.1), 132.5(d, J=6.3), 130.0,128.9,127.6(d, J=10.5)$, $119.9,110.5,104.1,104.0,95.4,88.9,88.4,86.9(d, J=6.4), 80.4$ $(d, J=10.1), 55.8,55.7,41.4(d, J=18.3), 35.9(d, J=18.4), 33.4$, $30.8,30.0,29.3,28.6(d, J=7.6), 28.0(d, J=8.9), 27.6(d, J=$ 14.5), 27.5, $26.8(d, J=14.2), 26.6,25.4,22.6,22.4,17.3 .{ }^{31} \mathrm{P}$ NMR $\left[\mathrm{CDCl}_{3}, 121 \mathrm{MHz}, 220 \mathrm{~K}, \delta(\mathrm{ppm})\right]: 39.06$.

\section{Refinement}

Crystal data, data collection and structure refinement details are summarized in Table 2. The hydrogen atoms were placed in calculated positions and refined using a riding model: $\mathrm{C}-\mathrm{H}$ $=0.95-1.00 \AA$ 品ith $U_{\text {iso }}(\mathrm{H})=1.5 U_{\text {eq }}(\mathrm{C}$-methyl $)$ and $1.2 \mathrm{U}_{\text {eq }}(\mathrm{C})$ for other $\mathrm{H}$ atoms.

The X-ray diffraction study was carried out on the 'Belok' beamline of the National Research Center Kurchatov Institute (Moscow, Russian Federation) using a Rayonix SX165 CCD detector.

A rather large number of reflections ( $c a$ 100) were omitted in the final cycles of refinement for the following reasons:
Table 2

Experimental details.

\begin{tabular}{ll}
\hline Crystal data & \\
Chemical formula & {$\left[\mathrm{RuCl}_{2}\left(\mathrm{C}_{10} \mathrm{H}_{14}\right)\left(\mathrm{C}_{26} \mathrm{H}_{35} \mathrm{O}_{2} \mathrm{P}\right)\right]$} \\
$M_{\mathrm{r}}$ & 716.69 \\
Crystal system, space group & Monoclinic, $P 2_{1} / c$ \\
Temperature $(\mathrm{K})$ & 100 \\
$a, b, c(\AA)$ & $19.790(4), 19.950(4), 20.225(4)$ \\
$\beta\left({ }^{\circ}\right)$ & $118.17(3)$ \\
$V\left(\AA^{3}\right)$ & $7039(3)$ \\
$Z$ & 8 \\
Radiation type & Synchrotron, $\lambda=0.96260 \AA$ \\
$\mu\left(\mathrm{mm}^{-1}\right)$ & 1.52 \\
Crystal size $(\mathrm{mm})$ & $0.20 \times 0.15 \times 0.10$ \\
& \\
Data collection & Rayonix SX165 CCD \\
Diffractometer & Multi-scan $(S C A L A ;$ Evans, 2006) \\
Absorption correction & $0.730,0.850$ \\
$T_{\min }, T_{\max }$ & $85347,15331,11898$ \\
No. of measured, independent and & \\
$\quad$ observed $[I>2 \sigma(I)]$ reflections & 0.080 \\
$R_{\text {int }}$ & 0.646 \\
(sin $\theta / \lambda)_{\text {max }}\left(\AA^{-1}\right)$ & \\
Refinement & \\
$R\left[F^{2}>2 \sigma\left(F^{2}\right)\right], w R\left(F^{2}\right), S$ & $0.059,0.160,1.07$ \\
No. of reflections & 15331 \\
No. of parameters & 768 \\
$H$-atom treatment & $\mathrm{H}$-atom parameters constrained \\
$\Delta \rho_{\text {max }}, \Delta \rho_{\text {min }}\left(\mathrm{e} \AA^{-3}\right)$ & $1.92,-1.77$ \\
\hline
\end{tabular}

Computer programs: MARCCD (Doyle, 2011), iMosflm (Battye et al., 2011), SHELXT (Sheldrick, 2015a), SHELXL2014 (Sheldrick, 2015b), SHELXTL (Sheldrick, 2008), Mercury (Macrae et al., 2008), PLATON (Spek, 2009) and publCIF(Westrip, 2010).

(1) In order to achieve better $I / \sigma$ statistics for high-angle reflections we selected exposure times to allow a small fraction of intensity overloads in the low-angle part of the detector. These low-angle reflections with imprecisely measured intensities were excluded from the final cycles of refinement.

2) In the present setup of the synchrotron diffractometer, the low-temperature device eclipses a small region of the image-plate detector near the high-angle limit. This small shadowed region was not masked during integration of the diffraction frames, which erroneously resulted in zero intensity of some reflections.

3) The quality of the single crystal chosen for the diffraction experiment was not perfect. Some systematic intensity distortions may be due to extinction and defects present in the crystal.

\section{Funding information}

This work was supported in part by the RUDN University Program (No. 5-100). Synchrotron radiation-based singlecrystal X-ray diffraction measurements were performed at the unique scientific facility Kurchatov Synchrotron Radiation Source supported by the Ministry of Education and Science of the Russian Federation (project code RFMEFI61917X0007).

\section{References}

Afanasyev, O. I., Tsygankov, A. A., Usanov, D. L., Perekalin, D. S., Samoylova, A. D. \& Chusov, D. (2017). Synthesis, 49, 2640-2651. 
Afanasyev, O. I., Tsygankov, A. A., Usanov, D. L., Perekalin, D. S., Shvydkiy, N. V., Maleev, V. I., Kudinov, A. R. \& Chusov, D. (2016). ACS Catal. 6, 2043-2046.

Aznar, R., Grabulosa, A., Mannu, A., Muller, G., Sainz, D., Moreno, V., Font-Bardia, M., Calvet, T. \& Lorenzo, J. (2013). Organometallics, 32, 2344-2362.

Baraut, J., Massard, A., Chotard, F., Bodio, E., Picquet, M., Richard, P., Borguet, Y., Nicks, F., Demonceau, A. \& Le Gendre, P. (2015). Eur. J. Inorg. Chem. 2015, 2671-2682.

Battye, T. G. G., Kontogiannis, L., Johnson, O., Powell, H. R. \& Leslie, A. G. W. (2011). Acta Cryst. D67, 271-281.

Cerón-Camacho, R., Gómez-Benítez, V., Le Lagadec, R., MoralesMorales, D. \& Toscano, R. A. (2006). J. Mol. Catal. A Chem. 247, 124-129.

Chuklin, P., Chalermpanaphan, V., Nhukeaw, T., Saithong, S., Chainok, K., Phongpaichit, S., Ratanaphan, A. \& Leesakul, N. (2017). J. Organomet. Chem. 846, 242-250.

Chusov, D. \& List, B. (2014). Angew. Chem. Int. Ed. 53, 51995201.

Clavero, P., Grabulosa, A., Rocamora, M., Muller, G. \& Font-Bardia, M. (2016). Dalton Trans. 45, 8513-8531.

Díaz-Álvarez, A. E., Crochet, P., Zablocka, M., Duhayon, C., Cadierno, V., Gimeno, J. \& Majoral, J. P. (2006). Adv. Synth. Catal. 348, 1671-1679.

Doyle, R. A. (2011). MARCCD software manual. Rayonix L. L. C. Evanston, IL 60201 USA.

Evans, P. (2006). Acta Cryst. D62, 72-82.

Granville, S. L., Welch, G. C. \& Stephan, D. W. (2012). Inorg. Chem. 51, 4711-4721.
Kaithal, A., Chatterjee, B. \& Gunanathan, C. (2016). Org. Lett. 18, 3402-3405.

Kolesnikov, P. N., Yagafarov, N. Z., Usanov, D. L., Maleev, V. I. \& Chusov, D. (2015). Org. Lett. 17, 173-175.

Macrae, C. F., Bruno, I. J., Chisholm, J. A., Edgington, P. R., McCabe, P., Pidcock, E., Rodriguez-Monge, L., Taylor, R., van de Streek, J. \& Wood, P. A. (2008). J. Appl. Cryst. 41, 466-470.

Moldes, I., de la Encarnación, E., Ros, J., Alvarez-Larena, Á., \& Piniella, J. F. (1998). J. Organomet. Chem. 566, 165-174.

Molotkov, A. P., Vinogradov, M. M., Moskovets, A. P., Chusova, O., Timofeev, S. V., Fastovskiy, V. A., Nelyubina, Y. V., Pavlov, A. A., Chusov, D. A. \& Loginov, D. A. (2017). Eur. J. Inorg. Chem. 2, 111.

Moskovets, A. P., Usanov, D. L., Afanasyev, O. I., Fastovskiy, V. A., Molotkov, A. P., Muratov, K. M., Denisov, G. L., Zlotskii, S. S., Smol'yakov, A. F., Loginov, D. A. \& Chusov, D. (2017). Org. Biomol. Chem. 15, 6384-6387.

Muller, A. \& Davis, W. L. (2012). Acta Cryst. E68, m1446-m1447.

Nazarov, A. A., Hartinger, C. G. \& Dyson, P. J. (2014). J. Organomet. Chem. 751, 251-260.

Sheldrick, G. M. (2008). Acta Cryst. A64, 112-122.

Sheldrick, G. M. (2015a). Acta Cryst. A71, 3-8.

Sheldrick, G. M. (2015b). Acta Cryst. C71, 3-8.

Spek, A. L. (2009). Acta Cryst. D65, 148-155.

Therrien, B. (2009). Coord. Chem. Rev. 253, 493-519.

Westrip, S. P. (2010). J. Appl. Cryst. 43, 920-925.

Yagafarov, N. Z., Usanov, D. L., Moskovets, A. P., Kagramanov, N. D., Maleev, V. I. \& Chusov, D. (2015). ChemCatChem, 7, 25902593. 


\section{supporting information}

Acta Cryst. (2018). E74, 487-491 [https://doi.org/10.1107/S2056989018003821]

Synthesis, crystal structure and catalytic activity in reductive amination of dichlorido( $\eta^{6}$-p-cymene)(2'-dicyclohexylphosphanyl-2,6-dimethoxybiphenyl$\kappa P$ )ruthenium(II)

Maria Makarova, Alexey A. Tsygankov, Olga Chusova, Ivan V. Linko, Pavel V. Dorovatovskii and Yan V. Zubavichus

Computing details

Data collection: MARCCD (Doyle, 2011); cell refinement: iMosflm (Battye et al., 2011); data reduction: iMosflm (Battye et al., 2011); program(s) used to solve structure: SHELXT (Sheldrick, 2015a); program(s) used to refine structure:

SHELXL2014 (Sheldrick, 2015b); molecular graphics: SHELXTL (Sheldrick, 2008) and Mercury (Macrae et al., 2008); software used to prepare material for publication: SHELXL (Sheldrick, 2015b), PLATON (Spek, 2009) and publCIF(Westrip, 2010).

Dichlorido( $\eta^{6}-p$-cymene) (2'-dicyclohexylphosphanyl-2,6-dimethoxybiphenyl- $\left.\kappa P\right)$ ruthenium(II)

Crystal data

$\left[\mathrm{RuCl}_{2}\left(\mathrm{C}_{10} \mathrm{H}_{14}\right)\left(\mathrm{C}_{26} \mathrm{H}_{35} \mathrm{O}_{2} \mathrm{P}\right)\right]$

$M_{r}=716.69$

Monoclinic, $P 2_{1} / c$

$a=19.790(4) \AA$

$b=19.950(4) \AA$

$c=20.225(4) \AA$

$\beta=118.17(3)^{\circ}$

$V=7039(3) \AA^{3}$

$Z=8$

$F(000)=2992$

$D_{\mathrm{x}}=1.353 \mathrm{Mg} \mathrm{m}^{-3}$

Synchrotron radiation, $\lambda=0.96260 \AA$

Cell parameters from 600 reflections

$\theta=3.2-35.0^{\circ}$

$\mu=1.52 \mathrm{~mm}^{-1}$

$T=100 \mathrm{~K}$

Prism, dark-orange

$0.20 \times 0.15 \times 0.10 \mathrm{~mm}$

Data collection

Rayonix SX165 CCD

diffractometer

$\varphi$ scan

Absorption correction: multi-scan

(SCALA; Evans, 2006)

$T_{\text {min }}=0.730, T_{\max }=0.850$

85347 measured reflections

15331 independent reflections

11898 reflections with $I>2 \sigma(I)$

$R_{\text {int }}=0.080$

$\theta_{\max }=38.4^{\circ}, \theta_{\min }=3.2^{\circ}$

$h=-25 \rightarrow 23$

$k=-25 \rightarrow 25$

$l=-26 \rightarrow 24$

Refinement

Refinement on $F^{2}$

Least-squares matrix: full

$R\left[F^{2}>2 \sigma\left(F^{2}\right)\right]=0.059$

$w R\left(F^{2}\right)=0.160$

$S=1.07$

15331 reflections

768 parameters

0 restraints

Primary atom site location: structure-invariant direct methods

Secondary atom site location: difference Fourier map 
Hydrogen site location: inferred from neighbouring sites

$\mathrm{H}$-atom parameters constrained

$w=1 /\left[\sigma^{2}\left(F_{\mathrm{o}}^{2}\right)+(0.0738 P)^{2}+8.8 P\right]$

where $P=\left(F_{\mathrm{o}}{ }^{2}+2 F_{\mathrm{c}}{ }^{2}\right) / 3$

$(\Delta / \sigma)_{\max }=0.001$
$\Delta \rho_{\max }=1.92 \mathrm{e} \AA^{-3}$

$\Delta \rho_{\min }=-1.77$ e $\AA^{-3}$

Extinction correction: SHELXL2014

(Sheldrick, 2015b),

$\mathrm{Fc}^{*}=\mathrm{kFc}\left[1+0.001 \mathrm{xFc}^{2} \lambda^{3} / \sin (2 \theta)\right]^{-1 / 4}$

Extinction coefficient: 0.0012 (1)

\section{Special details}

Geometry. All esds (except the esd in the dihedral angle between two 1.s. planes) are estimated using the full covariance matrix. The cell esds are taken into account individually in the estimation of esds in distances, angles and torsion angles; correlations between esds in cell parameters are only used when they are defined by crystal symmetry. An approximate (isotropic) treatment of cell esds is used for estimating esds involving 1.s. planes.

Fractional atomic coordinates and isotropic or equivalent isotropic displacement parameters $\left(\AA^{2}\right)$

\begin{tabular}{|c|c|c|c|c|}
\hline & $x$ & $y$ & $z$ & $U_{\text {iso }} * / U_{\text {eq }}$ \\
\hline Ru1 & $0.39465(2)$ & $0.34941(2)$ & $0.26968(2)$ & $0.02059(11)$ \\
\hline $\mathrm{Cl1}$ & $0.37597(5)$ & $0.31613(4)$ & $0.37623(5)$ & $0.0248(2)$ \\
\hline $\mathrm{Cl} 2$ & $0.30004(5)$ & $0.26463(4)$ & $0.19505(5)$ & $0.0264(2)$ \\
\hline P1 & $0.49089(5)$ & $0.26228(4)$ & $0.30732(5)$ & $0.0197(2)$ \\
\hline $\mathrm{O} 1$ & $0.70112(16)$ & $0.17532(12)$ & $0.34952(16)$ & $0.0293(6)$ \\
\hline $\mathrm{O} 2$ & $0.63517(16)$ & $0.39635(13)$ & $0.25883(17)$ & $0.0329(6)$ \\
\hline $\mathrm{C} 1$ & $0.3772(2)$ & $0.41199(16)$ & $0.1694(2)$ & $0.0237(8)$ \\
\hline $\mathrm{C} 2$ & $0.3194(2)$ & $0.42974(17)$ & $0.1905(2)$ & $0.0275(9)$ \\
\hline $\mathrm{H} 2$ & 0.2668 & 0.4275 & 0.1540 & $0.033^{*}$ \\
\hline $\mathrm{C} 3$ & 0.3389 & $0.45023(17)$ & $0.2638(3)$ & $0.0325(10)$ \\
\hline H3 & 0.2996 & 0.4630 & 0.2756 & $0.039^{*}$ \\
\hline $\mathrm{C} 4$ & $0.4174(3)$ & $0.45222(16)$ & $0.3212(2)$ & $0.0322(10)$ \\
\hline $\mathrm{C} 5$ & $0.4762(2)$ & $0.43513(17)$ & $0.3016(2)$ & $0.0266(8)$ \\
\hline H5 & 0.5287 & 0.4375 & 0.3382 & $0.032 *$ \\
\hline C6 & $0.4548(2)$ & $0.41436(16)$ & $0.2261(2)$ & $0.0255(8)$ \\
\hline H6 & 0.4939 & 0.4018 & 0.2139 & $0.031 *$ \\
\hline $\mathrm{C} 7$ & $0.3581(3)$ & $0.39964(19)$ & $0.0878(2)$ & $0.0339(9)$ \\
\hline $\mathrm{H} 7$ & 0.4004 & 0.3720 & 0.0882 & $0.041^{*}$ \\
\hline $\mathrm{C} 8$ & 0.3595 & 0.46815 (19) & $0.0540(2)$ & $0.0371(10)$ \\
\hline H8A & 0.3200 & 0.4971 & 0.0549 & $0.056^{*}$ \\
\hline H8B & 0.3495 & 0.4622 & 0.0021 & $0.056^{*}$ \\
\hline $\mathrm{H} 8 \mathrm{C}$ & 0.4099 & 0.4889 & 0.0834 & $0.056^{*}$ \\
\hline C9 & $0.2837(4)$ & 0.3638 & 0.0395 & $0.075(2)$ \\
\hline H9A & 0.2857 & 0.3185 & 0.0590 & $0.113^{*}$ \\
\hline H9B & 0.2757 & 0.3611 & -0.0121 & $0.113^{*}$ \\
\hline $\mathrm{H} 9 \mathrm{C}$ & 0.2412 & 0.3886 & 0.0401 & $0.113^{*}$ \\
\hline $\mathrm{C} 10$ & 0.4381 & $0.4735(2)$ & 0.4003 & $0.0489(13)$ \\
\hline H10A & 0.4028 & 0.4525 & 0.4154 & $0.073 *$ \\
\hline H10B & 0.4342 & 0.5224 & 0.4021 & $0.073 *$ \\
\hline $\mathrm{H} 10 \mathrm{C}$ & 0.4907 & 0.4595 & 0.4345 & $0.073 *$ \\
\hline C11 & $0.5865(2)$ & $0.30029(17)$ & $0.3731(2)$ & $0.0235(8)$ \\
\hline $\mathrm{C} 12$ & $0.6558(2)$ & $0.30373(18)$ & $0.3684(2)$ & $0.0255(8)$ \\
\hline $\mathrm{C} 13$ & $0.7216(2)$ & $0.3298(2)$ & $0.4312(2)$ & $0.0331(9)$ \\
\hline
\end{tabular}




\begin{tabular}{|c|c|c|c|c|}
\hline H13 & 0.7687 & 0.3309 & 0.4294 & $0.040^{*}$ \\
\hline $\mathrm{C} 14$ & 0.7204 & $0.3538(2)$ & 0.4953 & $0.0359(10)$ \\
\hline H14 & 0.7659 & 0.3702 & 0.5365 & $0.043 *$ \\
\hline $\mathrm{C} 15$ & $0.6510(2)$ & $0.35352(19)$ & $0.4982(2)$ & $0.0315(9)$ \\
\hline H15 & 0.6487 & 0.3708 & 0.5409 & $0.038^{*}$ \\
\hline $\mathrm{C} 16$ & $0.5856(2)$ & $0.32755(18)$ & $0.4379(2)$ & $0.0269(8)$ \\
\hline H16 & 0.5387 & 0.3280 & 0.4401 & $0.032 *$ \\
\hline C17 & $0.6690(2)$ & $0.28524(18)$ & $0.3027(2)$ & $0.0249(8)$ \\
\hline C18 & $0.6972(2)$ & $0.22167(18)$ & $0.2973(2)$ & $0.0258(8)$ \\
\hline C19 & $0.7185(2)$ & $0.2073(2)$ & $0.2411(2)$ & $0.0316(9)$ \\
\hline H19 & 0.7372 & 0.1642 & 0.2380 & $0.038 *$ \\
\hline $\mathrm{C} 20$ & $0.7113(3)$ & $0.2582(2)$ & 0.1901 & $0.0359(10)$ \\
\hline $\mathrm{H} 20$ & 0.7253 & 0.2491 & 0.1521 & $0.043^{*}$ \\
\hline $\mathrm{C} 21$ & $0.6841(2)$ & $0.3218(2)$ & $0.1940(2)$ & $0.0335(9)$ \\
\hline $\mathrm{H} 21$ & 0.6800 & 0.3557 & 0.1594 & $0.040^{*}$ \\
\hline $\mathrm{C} 22$ & $0.6627(2)$ & 0.33488 (19) & $0.2500(2)$ & $0.0279(8)$ \\
\hline $\mathrm{C} 23$ & $0.7143(3)$ & 0.10637 (19) & $0.3367(3)$ & $0.0392(11)$ \\
\hline $\mathrm{H} 23 \mathrm{~A}$ & 0.6770 & 0.0932 & 0.2856 & $0.059 *$ \\
\hline $\mathrm{H} 23 \mathrm{~B}$ & 0.7086 & 0.0774 & 0.3728 & $0.059 *$ \\
\hline $\mathrm{H} 23 \mathrm{C}$ & 0.7663 & 0.1018 & 0.3429 & $0.059 *$ \\
\hline $\mathrm{C} 24$ & 0.6260 & $0.4486(2)$ & $0.2060(3)$ & 0.0403 (11) \\
\hline $\mathrm{H} 24 \mathrm{~A}$ & 0.5901 & 0.4337 & 0.1551 & $0.060 *$ \\
\hline H24B & 0.6757 & 0.4586 & 0.2087 & $0.060 *$ \\
\hline $\mathrm{H} 24 \mathrm{C}$ & 0.6059 & 0.4891 & 0.2181 & $0.060^{*}$ \\
\hline $\mathrm{C} 25$ & $0.4883(2)$ & $0.19855(17)$ & $0.3755(2)$ & $0.0226(8)$ \\
\hline $\mathrm{H} 25$ & 0.4913 & 0.2267 & 0.4177 & $0.027 *$ \\
\hline $\mathrm{C} 26$ & $0.5590(2)$ & $0.15215(17)$ & $0.4139(2)$ & $0.0257(8)$ \\
\hline $\mathrm{H} 26 \mathrm{~A}$ & 0.5564 & 0.1164 & 0.3789 & $0.031 *$ \\
\hline $\mathrm{H} 26 \mathrm{~B}$ & 0.6066 & 0.1782 & 0.4288 & $0.031^{*}$ \\
\hline $\mathrm{C} 27$ & $0.5592(2)$ & 0.12113 (19) & $0.4837(2)$ & $0.0310(9)$ \\
\hline $\mathrm{H} 27 \mathrm{~A}$ & 0.5674 & 0.1570 & 0.5207 & $0.037^{*}$ \\
\hline $\mathrm{H} 27 \mathrm{~B}$ & 0.6022 & 0.0890 & 0.5072 & $0.037^{*}$ \\
\hline C28 & $0.4830(2)$ & 0.08444 (19) & $0.4640(2)$ & $0.0325(9)$ \\
\hline $\mathrm{H} 28 \mathrm{~A}$ & 0.4794 & 0.0439 & 0.4343 & $0.039 *$ \\
\hline $\mathrm{H} 28 \mathrm{~B}$ & 0.4835 & 0.0698 & 0.5110 & $0.039 *$ \\
\hline C29 & $0.4117(2)$ & 0.12857 (19) & $0.4191(2)$ & $0.0309(9)$ \\
\hline H29A & 0.3649 & 0.1012 & 0.4032 & $0.037 *$ \\
\hline H29B & 0.4105 & 0.1652 & 0.4516 & $0.037 *$ \\
\hline C30 & $0.4126(2)$ & $0.15879(17)$ & $0.3496(2)$ & $0.0248(8)$ \\
\hline $\mathrm{H} 30 \mathrm{~A}$ & 0.3681 & 0.1890 & 0.3232 & $0.030 *$ \\
\hline H30B & 0.4092 & 0.1226 & 0.3146 & $0.030 *$ \\
\hline C31 & $0.5014(2)$ & $0.21346(17)$ & $0.2328(2)$ & $0.0235(8)$ \\
\hline H31 & 0.5564 & 0.1999 & 0.2545 & $0.028 *$ \\
\hline C32 & $0.4824(2)$ & $0.25709(17)$ & $0.1633(2)$ & $0.0253(8)$ \\
\hline $\mathrm{H} 32 \mathrm{~A}$ & 0.5147 & 0.2979 & 0.1791 & $0.030 *$ \\
\hline H32B & 0.4281 & 0.2713 & 0.1407 & $0.030 *$ \\
\hline C33 & $0.4959(3)$ & $0.2195(2)$ & $0.1038(2)$ & $0.0327(9)$ \\
\hline H33A & 0.4813 & 0.2487 & 0.0596 & $0.039 *$ \\
\hline
\end{tabular}




\begin{tabular}{|c|c|c|c|c|}
\hline H33B & 0.5510 & 0.2085 & 0.1248 & $0.039 *$ \\
\hline C34 & $0.4483(3)$ & $0.15464(19)$ & $0.0796(2)$ & $0.0358(10)$ \\
\hline $\mathrm{H} 34 \mathrm{~A}$ & 0.3931 & 0.1659 & 0.0533 & $0.043^{*}$ \\
\hline H34B & 0.4606 & 0.1294 & 0.0444 & $0.043^{*}$ \\
\hline C35 & $0.4661(3)$ & $0.11097(19)$ & $0.1486(2)$ & $0.0325(9)$ \\
\hline $\mathrm{H} 35 \mathrm{~A}$ & 0.5202 & 0.0962 & 0.1715 & $0.039 *$ \\
\hline H35B & 0.4334 & 0.0704 & 0.1322 & $0.039 *$ \\
\hline C36 & $0.4525(2)$ & $0.14818(17)$ & $0.2079(2)$ & $0.0264(8)$ \\
\hline $\mathrm{H} 36 \mathrm{~A}$ & 0.4666 & 0.1188 & 0.2519 & $0.032 *$ \\
\hline H36B & 0.3975 & 0.1596 & 0.1868 & $0.032 *$ \\
\hline Ru2 & $0.11771(2)$ & $0.45313(2)$ & $0.25706(2)$ & $0.02841(11)$ \\
\hline $\mathrm{Cl} 3$ & $0.20723(6)$ & $0.54512(4)$ & $0.31822(6)$ & $0.0308(2)$ \\
\hline $\mathrm{Cl} 4$ & $0.12802(6)$ & $0.48211(5)$ & $0.14440(6)$ & $0.0320(2)$ \\
\hline P2 & $0.01492(6)$ & $0.53415(5)$ & $0.21645(6)$ & $0.0281(2)$ \\
\hline $\mathrm{O} 3$ & $-0.19526(16)$ & $0.61509(13)$ & $0.16977(16)$ & $0.0319(6)$ \\
\hline $\mathrm{O} 4$ & $-0.12390(17)$ & $0.40226(15)$ & $0.28418(18)$ & $0.0409(7)$ \\
\hline C37 & $0.1490(3)$ & 0.39587 (19) & $0.3650(3)$ & $0.0339(10)$ \\
\hline $\mathrm{C} 38$ & $0.2032(2)$ & $0.37920(18)$ & $0.3386(2)$ & $0.0293(9)$ \\
\hline H38 & 0.2564 & 0.3846 & 0.3716 & $0.035^{*}$ \\
\hline C39 & $0.1800(3)$ & $0.35547(18)$ & 0.2664 (3) & $0.0356(10)$ \\
\hline H39 & 0.2175 & 0.3443 & 0.2515 & $0.043^{*}$ \\
\hline $\mathrm{C} 40$ & 0.0995 (3) & $0.34759(19)$ & 0.2137 (3) & $0.0403(11)$ \\
\hline $\mathrm{C} 41$ & $0.0448(3)$ & $0.3627(2)$ & $0.2383(3)$ & $0.0414(11)$ \\
\hline H41 & -0.0083 & 0.3568 & 0.2053 & $0.050^{*}$ \\
\hline $\mathrm{C} 42$ & $0.0702(3)$ & $0.38710(19)$ & $0.3141(3)$ & $0.0375(11)$ \\
\hline H42 & 0.0332 & 0.3974 & 0.3298 & $0.045^{*}$ \\
\hline $\mathrm{C} 43$ & $0.1769(3)$ & $0.4108(2)$ & $0.4482(3)$ & $0.0438(12)$ \\
\hline $\mathrm{H} 43$ & 0.1349 & 0.4347 & 0.4528 & $0.053^{*}$ \\
\hline $\mathrm{C} 44$ & 0.1908 (4) & $0.3431(2)$ & 0.4895 (3) & $0.0590(15)$ \\
\hline $\mathrm{H} 44 \mathrm{~A}$ & 0.1430 & 0.3174 & 0.4684 & $0.088^{*}$ \\
\hline H44B & 0.2084 & 0.3512 & 0.5430 & $0.088^{*}$ \\
\hline $\mathrm{H} 44 \mathrm{C}$ & 0.2300 & 0.3178 & 0.4835 & $0.088^{*}$ \\
\hline $\mathrm{C} 45$ & 0.2497 (4) & $0.4544(2)$ & 0.4859 (3) & $0.0578(15)$ \\
\hline $\mathrm{H} 45 \mathrm{~A}$ & 0.2920 & 0.4317 & 0.4828 & $0.087 *$ \\
\hline H45B & 0.2633 & 0.4616 & 0.5386 & $0.087^{*}$ \\
\hline $\mathrm{H} 45 \mathrm{C}$ & 0.2400 & 0.4978 & 0.4601 & $0.087^{*}$ \\
\hline C46 & 0.0759 (3) & $0.3237(2)$ & 0.1349 (3) & $0.0546(14)$ \\
\hline H46A & 0.0201 & 0.3271 & 0.1047 & $0.082 *$ \\
\hline H46B & 0.0916 & 0.2770 & 0.1364 & $0.082 *$ \\
\hline $\mathrm{H} 46 \mathrm{C}$ & 0.1006 & 0.3516 & 0.1126 & $0.082^{*}$ \\
\hline $\mathrm{C} 47$ & $-0.0779(2)$ & $0.48924(18)$ & $0.1583(2)$ & $0.0298(9)$ \\
\hline $\mathrm{C} 48$ & $-0.1455(2)$ & $0.48246(18)$ & $0.1672(2)$ & $0.0281(8)$ \\
\hline C49 & $-0.2067(2)$ & 0.44357 (19) & $0.1142(2)$ & $0.0315(9)$ \\
\hline H49 & -0.2513 & 0.4387 & 0.1202 & $0.038^{*}$ \\
\hline $\mathrm{C} 50$ & $-0.2050(2)$ & 0.41191 (19) & $0.0536(2)$ & $0.0336(9)$ \\
\hline H50 & -0.2474 & 0.3862 & 0.0192 & $0.040^{*}$ \\
\hline $\mathrm{C} 51$ & $-0.1395(2)$ & 0.4188 (2) & 0.0443 (3) & $0.0364(10)$ \\
\hline H51 & -0.1372 & 0.3979 & 0.0033 & $0.044 *$ \\
\hline
\end{tabular}




\begin{tabular}{|c|c|c|c|c|}
\hline C52 & $-0.0778(2)$ & 0.45664 (19) & $0.0960(3)$ & $0.0339(10)$ \\
\hline H52 & -0.0337 & 0.4607 & 0.0892 & $0.041^{*}$ \\
\hline C53 & $-0.1596(2)$ & $0.50943(19)$ & $0.2295(2)$ & $0.0295(9)$ \\
\hline C54 & $-0.1899(2)$ & $0.5741(2)$ & $0.2270(2)$ & $0.0300(9)$ \\
\hline C55 & $-0.2122(2)$ & $0.5943(2)$ & $0.2811(3)$ & $0.0364(10)$ \\
\hline H55 & -0.2316 & 0.6381 & 0.2801 & $0.044^{*}$ \\
\hline C56 & -0.2050 & $0.5477(2)$ & $0.3363(3)$ & 0.0449 (12) \\
\hline H56 & -0.2207 & 0.5605 & 0.3721 & $0.054^{*}$ \\
\hline C57 & $-0.1756(3)$ & $0.4836(2)$ & $0.3403(3)$ & $0.0434(11)$ \\
\hline H57 & -0.1705 & 0.4533 & 0.3787 & $0.052 *$ \\
\hline C58 & $-0.1536(2)$ & $0.4648(2)$ & $0.2865(3)$ & $0.0345(10)$ \\
\hline C59 & -0.2050 & 0.68606 (19) & $0.1768(3)$ & $0.0354(10)$ \\
\hline H59A & -0.1990 & 0.7103 & 0.1377 & $0.053^{*}$ \\
\hline H59B & -0.1662 & 0.7016 & 0.2262 & $0.053^{*}$ \\
\hline H59C & -0.2562 & 0.6944 & 0.1711 & $0.053^{*}$ \\
\hline C60 & $-0.1214(3)$ & $0.3521(2)$ & $0.3364(3)$ & 0.0505 (13) \\
\hline H60A & -0.1026 & 0.3099 & 0.3265 & $0.076^{*}$ \\
\hline H60B & -0.1730 & 0.3455 & 0.3305 & $0.076^{*}$ \\
\hline H60C & -0.0870 & 0.3669 & 0.3877 & $0.076^{*}$ \\
\hline C61 & $0.0064(2)$ & $0.59682(19)$ & $0.1432(3)$ & $0.0315(9)$ \\
\hline H61 & -0.0010 & 0.5687 & 0.0994 & $0.038^{*}$ \\
\hline C62 & $-0.0677(2)$ & $0.6395(2)$ & $0.1133(3)$ & $0.0362(10)$ \\
\hline H62A & -0.1119 & 0.6102 & 0.1032 & $0.043^{*}$ \\
\hline H62B & -0.0627 & 0.6730 & 0.1515 & $0.043^{*}$ \\
\hline C63 & $-0.0813(3)$ & $0.6754(2)$ & $0.0408(3)$ & $0.0420(11)$ \\
\hline H63A & -0.1278 & 0.7036 & 0.0226 & $0.050 *$ \\
\hline H63B & -0.0900 & 0.6417 & 0.0017 & $0.050 *$ \\
\hline C64 & $-0.0122(3)$ & $0.7195(2)$ & $0.0542(3)$ & $0.0448(11)$ \\
\hline H64A & -0.0213 & 0.7404 & 0.0063 & $0.054^{*}$ \\
\hline H64B & -0.0066 & 0.7559 & 0.0898 & $0.054^{*}$ \\
\hline C65 & $0.0622(3)$ & $0.6782(2)$ & $0.0862(3)$ & $0.0376(10)$ \\
\hline H65A & 0.1058 & 0.7082 & 0.0966 & $0.045^{*}$ \\
\hline H65B & 0.0585 & 0.6448 & 0.0484 & $0.045 *$ \\
\hline C66 & $0.0772(2)$ & $0.64132(19)$ & $0.1594(3)$ & $0.0343(10)$ \\
\hline H66A & 0.0856 & 0.6744 & 0.1991 & $0.041 *$ \\
\hline H66B & 0.1236 & 0.6131 & 0.1771 & $0.041^{*}$ \\
\hline C67 & $0.0060(2)$ & $0.58350(19)$ & $0.2913(2)$ & $0.0314(9)$ \\
\hline H67 & -0.0488 & 0.5978 & 0.2701 & $0.038^{*}$ \\
\hline C68 & $0.0244(3)$ & $0.5391(2)$ & $0.3605(3)$ & $0.0365(10)$ \\
\hline H68A & 0.0785 & 0.5242 & 0.3830 & $0.044 *$ \\
\hline H68B & -0.0086 & 0.4988 & 0.3444 & $0.044^{*}$ \\
\hline C69 & $0.0115(3)$ & $0.5774(2)$ & $0.4203(3)$ & $0.0453(11)$ \\
\hline H69A & -0.0433 & 0.5896 & 0.3993 & $0.054^{*}$ \\
\hline H69B & 0.0252 & 0.5481 & 0.4644 & $0.054^{*}$ \\
\hline $\mathrm{C} 70$ & $0.0610(3)$ & $0.6411(3)$ & $0.4448(3)$ & $0.0511(13)$ \\
\hline H70A & 0.0497 & 0.6667 & 0.4803 & $0.061^{*}$ \\
\hline H70B & 0.1159 & 0.6284 & 0.4709 & $0.061^{*}$ \\
\hline C71 & $0.0452(3)$ & $0.6850(2)$ & $0.3772(3)$ & $0.0515(13)$ \\
\hline
\end{tabular}




$\begin{array}{lllll}\text { H71A } & 0.0801 & 0.7241 & 0.3944 & 0.062^{*} \\ \text { H71B } & -0.0080 & 0.7020 & 0.3552 & 0.062^{*} \\ \text { C72 } & 0.0558(3) & 0.6480(2) & 0.3162(3) & 0.0380(10) \\ \text { H72A } & 0.1105 & 0.6359 & 0.3357 & 0.046^{*} \\ \text { H72B } & 0.0410 & 0.6778 & 0.2724 & 0.046^{*}\end{array}$

Atomic displacement parameters $\left(\AA^{2}\right)$

\begin{tabular}{|c|c|c|c|c|c|c|}
\hline & $U^{11}$ & $U^{22}$ & $U^{33}$ & $U^{12}$ & $U^{13}$ & $U^{23}$ \\
\hline Ru1 & $0.0215(2)$ & $0.01744(15)$ & $0.02680(18)$ & $0.00103(10)$ & $0.01469(15)$ & $0.00164(10)$ \\
\hline $\mathrm{Cl1}$ & $0.0269(5)$ & $0.0244(4)$ & $0.0302(5)$ & $0.0029(3)$ & $0.0192(4)$ & $0.0034(3)$ \\
\hline $\mathrm{Cl} 2$ & $0.0258(5)$ & $0.0221(4)$ & $0.0314(5)$ & $-0.0006(3)$ & $0.0137(4)$ & $0.0002(3)$ \\
\hline $\mathrm{P} 1$ & $0.0193(5)$ & $0.0175(4)$ & $0.0245(5)$ & $0.0013(3)$ & $0.0122(4)$ & $0.0013(3)$ \\
\hline $\mathrm{O} 1$ & $0.0334(17)$ & $0.0230(13)$ & $0.0375(16)$ & $0.0036(11)$ & $0.0216(14)$ & $0.0033(11)$ \\
\hline $\mathrm{O} 2$ & $0.0303(17)$ & $0.0280(14)$ & $0.0454(18)$ & $0.0040(12)$ & $0.0220(15)$ & $0.0080(12)$ \\
\hline $\mathrm{C} 1$ & $0.031(2)$ & $0.0181(16)$ & 0.0247 (19) & $0.0002(15)$ & $0.0151(18)$ & $0.0124(14)$ \\
\hline $\mathrm{C} 2$ & $0.026(2)$ & $0.0175(16)$ & $0.039(2)$ & $0.0127(15)$ & 0.0153 (19) & $0.0206(15)$ \\
\hline $\mathrm{C} 3$ & $0.041(3)$ & $0.0167(17)$ & $0.055(3)$ & $0.0120(16)$ & $0.036(3)$ & 0.0093 (16) \\
\hline $\mathrm{C} 4$ & $0.058(3)$ & $0.0095(16)$ & $0.036(2)$ & -0.0049 (16) & $0.029(2)$ & $-0.0083(14)$ \\
\hline $\mathrm{C} 5$ & $0.027(2)$ & $0.0149(16)$ & $0.033(2)$ & $-0.0127(15)$ & $0.0110(19)$ & $-0.0028(14)$ \\
\hline C6 & $0.029(2)$ & $0.0179(16)$ & $0.041(2)$ & $-0.0032(15)$ & $0.026(2)$ & $0.0066(15)$ \\
\hline $\mathrm{C} 7$ & $0.045(3)$ & $0.030(2)$ & 0.029 (2) & 0.0005 (18) & $0.020(2)$ & $0.0018(16)$ \\
\hline $\mathrm{C} 8$ & $0.057(3)$ & $0.029(2)$ & $0.036(2)$ & 0.0077 (19) & $0.031(2)$ & 0.0073 (17) \\
\hline C9 & $0.084(5)$ & $0.106(5)$ & $0.023(3)$ & $-0.053(4)$ & $0.014(3)$ & $-0.001(3)$ \\
\hline $\mathrm{C} 10$ & $0.086(4)$ & $0.031(2)$ & $0.045(3)$ & $-0.015(2)$ & $0.043(3)$ & -0.0092 (19) \\
\hline $\mathrm{C} 11$ & $0.023(2)$ & $0.0212(17)$ & $0.028(2)$ & $-0.0001(14)$ & $0.0136(18)$ & $-0.0002(14)$ \\
\hline $\mathrm{C} 12$ & $0.024(2)$ & $0.0229(18)$ & $0.033(2)$ & $0.0020(15)$ & 0.0155 (19) & $0.0021(15)$ \\
\hline $\mathrm{C} 13$ & $0.019(2)$ & $0.042(2)$ & $0.038(2)$ & $-0.0065(17)$ & $0.013(2)$ & $-0.0056(18)$ \\
\hline $\mathrm{C} 14$ & $0.026(3)$ & $0.042(2)$ & $0.033(2)$ & $-0.0061(18)$ & $0.009(2)$ & $-0.0090(18)$ \\
\hline $\mathrm{C} 15$ & $0.033(3)$ & $0.032(2)$ & $0.031(2)$ & $-0.0037(17)$ & $0.015(2)$ & $-0.0080(16)$ \\
\hline $\mathrm{C} 16$ & $0.022(2)$ & 0.0285 (19) & $0.032(2)$ & $0.0003(16)$ & 0.0147 (19) & $-0.0007(16)$ \\
\hline $\mathrm{C} 17$ & $0.024(2)$ & $0.0261(18)$ & $0.029(2)$ & $-0.0012(15)$ & $0.0156(18)$ & $0.0018(15)$ \\
\hline $\mathrm{C} 18$ & $0.020(2)$ & 0.0291 (19) & $0.031(2)$ & $-0.0022(15)$ & 0.0135 & $0.0022(15)$ \\
\hline C19 & $0.025(2)$ & $0.035(2)$ & $0.040(2)$ & 0.0033 (17) & $0.020(2)$ & $0.0004(17)$ \\
\hline $\mathrm{C} 20$ & $0.033(3)$ & $0.046(2)$ & 0.037 (2) & -0.0030 (19) & $0.024(2)$ & $-0.0013(19)$ \\
\hline $\mathrm{C} 21$ & $0.033(3)$ & 0.039 (2) & $0.035(2)$ & $-0.0063(18)$ & $0.021(2)$ & $0.0060(18)$ \\
\hline $\mathrm{C} 22$ & $0.020(2)$ & 0.0284 (19) & $0.034(2)$ & $-0.0024(16)$ & 0.0126 (19) & 0.0013 (16) \\
\hline $\mathrm{C} 23$ & $0.047(3)$ & $0.027(2)$ & $0.054(3)$ & 0.0074 (19) & $0.032(3)$ & 0.0035 (19) \\
\hline $\mathrm{C} 24$ & $0.037(3)$ & $0.031(2)$ & $0.058(3)$ & $-0.0012(18)$ & $0.026(3)$ & 0.0140 (19) \\
\hline $\mathrm{C} 25$ & $0.023(2)$ & $0.0211(17)$ & $0.028(2)$ & $0.0020(14)$ & $0.0153(18)$ & 0.0023 (14) \\
\hline $\mathrm{C} 26$ & $0.024(2)$ & $0.0211(17)$ & $0.033(2)$ & $0.0013(15)$ & 0.0141 (19) & 0.0007 (14) \\
\hline $\mathrm{C} 27$ & $0.034(3)$ & 0.0260 (19) & $0.035(2)$ & $0.0056(17)$ & $0.018(2)$ & $0.0036(16)$ \\
\hline $\mathrm{C} 28$ & $0.040(3)$ & 0.0262 (19) & $0.035(2)$ & $0.0031(17)$ & $0.021(2)$ & $0.0091(16)$ \\
\hline $\mathrm{C} 29$ & $0.035(3)$ & 0.0265 (19) & $0.039(2)$ & $-0.0015(17)$ & $0.025(2)$ & $0.0033(16)$ \\
\hline $\mathrm{C} 30$ & $0.026(2)$ & $0.0222(17)$ & $0.029(2)$ & $0.0007(15)$ & $0.0156(18)$ & $0.0022(14)$ \\
\hline $\mathrm{C} 31$ & $0.022(2)$ & $0.0205(17)$ & $0.030(2)$ & $0.0032(14)$ & $0.0147(18)$ & $-0.0001(14)$ \\
\hline $\mathrm{C} 32$ & $0.030(2)$ & 0.0198 (17) & $0.031(2)$ & $0.0030(15)$ & 0.0182 (19) & $0.0006(14)$ \\
\hline C33 & $0.044(3)$ & $0.032(2)$ & $0.028(2)$ & $0.0043(18)$ & $0.023(2)$ & $0.0001(16)$ \\
\hline
\end{tabular}




\begin{tabular}{|c|c|c|c|c|c|c|}
\hline C34 & $0.046(3)$ & $0.032(2)$ & $0.028(2)$ & $0.0030(18)$ & $0.016(2)$ & $-0.0031(16)$ \\
\hline $\mathrm{C} 35$ & $0.043(3)$ & $0.0243(19)$ & $0.030(2)$ & 0.0005 (17) & $0.017(2)$ & $-0.0013(15)$ \\
\hline $\mathrm{C} 36$ & $0.028(2)$ & $0.0201(17)$ & $0.031(2)$ & $0.0002(15)$ & $0.0135(19)$ & $0.0012(14)$ \\
\hline Ru2 & $0.0228(2)$ & $0.02222(17)$ & $0.0449(2)$ & $-0.00017(11)$ & $0.01981(17)$ & $-0.00099(12)$ \\
\hline $\mathrm{Cl} 3$ & $0.0262(6)$ & $0.0247(4)$ & $0.0429(6)$ & $-0.0014(4)$ & $0.0175(5)$ & $-0.0024(4)$ \\
\hline $\mathrm{Cl} 4$ & $0.0266(6)$ & $0.0308(5)$ & $0.0447(6)$ & $0.0016(4)$ & $0.0219(5)$ & $-0.0013(4)$ \\
\hline P2 & $0.0206(6)$ & $0.0250(5)$ & $0.0415(6)$ & $-0.0002(4)$ & $0.0170(5)$ & $-0.0031(4)$ \\
\hline $\mathrm{O} 3$ & $0.0325(17)$ & $0.0276(14)$ & $0.0412(17)$ & $0.0050(12)$ & $0.0221(15)$ & $-0.0003(12)$ \\
\hline $\mathrm{O} 4$ & $0.0315(18)$ & $0.0410(17)$ & $0.0512(19)$ & $0.0036(13)$ & $0.0204(16)$ & $0.0143(14)$ \\
\hline C37 & $0.039(3)$ & $0.0228(19)$ & $0.045(3)$ & $0.0032(17)$ & $0.024(2)$ & $0.0075(17)$ \\
\hline $\mathrm{C} 38$ & $0.023(2)$ & $0.0211(18)$ & $0.043(2)$ & $0.0086(15)$ & $0.015(2)$ & $0.0097(16)$ \\
\hline C39 & $0.037(3)$ & $0.0180(18)$ & $0.061(3)$ & $0.0073(16)$ & $0.031(3)$ & $0.0024(18)$ \\
\hline $\mathrm{C} 40$ & $0.051(3)$ & 0.0177 (19) & $0.056(3)$ & $-0.0084(18)$ & $0.028(3)$ & $-0.0107(18)$ \\
\hline C41 & $0.032(3)$ & $0.0228(19)$ & $0.069(3)$ & $-0.0147(18)$ & $0.024(3)$ & $-0.002(2)$ \\
\hline $\mathrm{C} 42$ & $0.036(3)$ & $0.0231(19)$ & $0.069(3)$ & $0.0000(17)$ & $0.037(3)$ & $0.0077(19)$ \\
\hline $\mathrm{C} 43$ & $0.055(3)$ & $0.031(2)$ & $0.058(3)$ & $0.007(2)$ & $0.037(3)$ & $0.004(2)$ \\
\hline $\mathrm{C} 44$ & $0.086(5)$ & $0.039(3)$ & $0.062(4)$ & $-0.004(3)$ & $0.043(3)$ & $0.009(2)$ \\
\hline $\mathrm{C} 45$ & $0.088(5)$ & $0.044(3)$ & $0.043(3)$ & $-0.015(3)$ & $0.033(3)$ & $0.000(2)$ \\
\hline $\mathrm{C} 46$ & $0.067(4)$ & $0.035(2)$ & $0.060(3)$ & $-0.013(2)$ & $0.029(3)$ & $-0.004(2)$ \\
\hline $\mathrm{C} 47$ & $0.024(2)$ & $0.0261(19)$ & $0.043(2)$ & $-0.0002(16)$ & $0.019(2)$ & $-0.0018(17)$ \\
\hline $\mathrm{C} 48$ & $0.023(2)$ & $0.0249(18)$ & $0.038(2)$ & $0.0020(15)$ & $0.0152(19)$ & $0.0018(16)$ \\
\hline C49 & $0.022(2)$ & $0.035(2)$ & $0.041(2)$ & $-0.0005(17)$ & $0.018(2)$ & $0.0035(17)$ \\
\hline $\mathrm{C} 50$ & $0.024(2)$ & $0.031(2)$ & $0.041(2)$ & $-0.0032(17)$ & $0.012(2)$ & $-0.0031(17)$ \\
\hline $\mathrm{C} 51$ & $0.030(3)$ & $0.035(2)$ & 0.045 & $0.0006(18)$ & $0.018(2)$ & $-0.0094(19)$ \\
\hline $\mathrm{C} 52$ & $0.023(2)$ & $0.033(2)$ & $0.051(3)$ & 0.0003 (17) & $0.022(2)$ & $-0.0068(18)$ \\
\hline C53 & $0.020(2)$ & $0.034(2)$ & $0.036(2)$ & $-0.0034(16)$ & $0.0142(19)$ & $0.0003(17)$ \\
\hline $\mathrm{C} 54$ & $0.022(2)$ & $0.038(2)$ & $0.033(2)$ & $-0.0036(17)$ & $0.0148(19)$ & $-0.0007(17)$ \\
\hline $\mathrm{C} 55$ & $0.026(2)$ & $0.045(2)$ & $0.043(3)$ & $-0.0018(18)$ & $0.020(2)$ & $-0.011(2)$ \\
\hline $\mathrm{C} 56$ & $0.042(3)$ & $0.062(3)$ & 0.040 & $-0.004(2)$ & 0.027 & $-0.006(2)$ \\
\hline C57 & $0.038(3)$ & $0.056(3)$ & $0.041(3)$ & -0.009 (2) & $0.023(2)$ & $0.000(2)$ \\
\hline $\mathrm{C} 58$ & $0.020(2)$ & $0.041(2)$ & $0.044(3)$ & $-0.0018(17)$ & $0.016(2)$ & 0.0069 (19) \\
\hline $\mathrm{C} 59$ & $0.031(3)$ & 0.0247 (19) & $0.054(3)$ & $0.0037(17)$ & $0.023(2)$ & $-0.0037(18)$ \\
\hline C60 & $0.041(3)$ & $0.048(3)$ & $0.055(3)$ & $0.001(2)$ & $0.017(3)$ & $0.022(2)$ \\
\hline C61 & $0.023(2)$ & $0.028(2)$ & $0.047(3)$ & $-0.0004(16)$ & $0.020(2)$ & $-0.0025(17)$ \\
\hline C62 & $0.025(2)$ & $0.031(2)$ & 0.055 & $0.0028(17)$ & $0.021(2)$ & 0.0000 (19) \\
\hline C63 & $0.030(3)$ & $0.032(2)$ & 0.060 & $0.0019(18)$ & $0.018(2)$ & $0.005(2)$ \\
\hline C64 & $0.039(3)$ & $0.033(2)$ & $0.061(3)$ & $-0.001(2)$ & $0.022(3)$ & $0.007(2)$ \\
\hline C65 & $0.031(3)$ & $0.031(2)$ & $0.054(3)$ & $-0.0008(18)$ & $0.023(2)$ & 0.0019 (19) \\
\hline C66 & $0.026(2)$ & $0.029(2)$ & $0.052(3)$ & $0.0005(17)$ & $0.022(2)$ & $-0.0025(18)$ \\
\hline C67 & $0.023(2)$ & $0.030(2)$ & $0.043(2)$ & $-0.0002(16)$ & $0.018(2)$ & $-0.0059(17)$ \\
\hline C68 & $0.025(2)$ & $0.042(2)$ & $0.041(3)$ & $0.0032(18)$ & $0.014(2)$ & $-0.0039(19)$ \\
\hline C69 & $0.034(3)$ & $0.060(3)$ & $0.046(3)$ & $0.002(2)$ & $0.022(2)$ & $-0.010(2)$ \\
\hline $\mathrm{C} 70$ & $0.034(3)$ & $0.070(3)$ & $0.054(3)$ & $-0.006(2)$ & $0.024(3)$ & $-0.027(3)$ \\
\hline C71 & $0.045(3)$ & $0.046(3)$ & $0.073(4)$ & -0.007 (2) & $0.036(3)$ & -0.027 \\
\hline C72 & $0.028(3)$ & $0.035(2)$ & $0.051(3)$ & $-0.0016(18)$ & $0.018(2)$ & $-0.0119(19)$ \\
\hline
\end{tabular}


Geometric parameters $\left(\AA,{ }^{\circ}\right)$

\begin{tabular}{|c|c|c|c|}
\hline $\mathrm{Ru} 1-\mathrm{C} 6$ & $2.205(3)$ & $\mathrm{Ru} 2-\mathrm{C} 41$ & $2.228(4)$ \\
\hline $\mathrm{Ru} 1-\mathrm{C} 5$ & $2.227(3)$ & $\mathrm{Ru} 2-\mathrm{C} 42$ & $2.230(4)$ \\
\hline $\mathrm{Ru} 1-\mathrm{C} 4$ & $2.247(3)$ & $\mathrm{Ru} 2-\mathrm{C} 40$ & $2.244(4)$ \\
\hline $\mathrm{Ru} 1-\mathrm{C} 2$ & $2.258(3)$ & $\mathrm{Ru} 2-\mathrm{C} 38$ & $2.263(4)$ \\
\hline $\mathrm{Ru} 1-\mathrm{C} 1$ & $2.266(3)$ & $\mathrm{Ru} 2-\mathrm{C} 39$ & $2.265(4)$ \\
\hline $\mathrm{Ru} 1-\mathrm{C} 3$ & $2.270(3)$ & $\mathrm{Ru} 2-\mathrm{C} 37$ & $2.276(4)$ \\
\hline $\mathrm{Ru} 1-\mathrm{P} 1$ & $2.4206(10)$ & $\mathrm{Ru} 2-\mathrm{P} 2$ & $2.4181(11)$ \\
\hline $\mathrm{Ru} 1-\mathrm{Cl1}$ & $2.4441(10)$ & $\mathrm{Ru} 2-\mathrm{Cl} 3$ & $2.4426(11)$ \\
\hline $\mathrm{Ru} 1-\mathrm{Cl} 2$ & $2.4445(11)$ & $\mathrm{Ru} 2-\mathrm{Cl} 4$ & $2.4508(11)$ \\
\hline $\mathrm{P} 1-\mathrm{C} 11$ & $1.884(4)$ & $\mathrm{P} 2-\mathrm{C} 47$ & $1.877(4)$ \\
\hline $\mathrm{P} 1-\mathrm{C} 31$ & $1.886(4)$ & $\mathrm{P} 2-\mathrm{C} 67$ & $1.883(4)$ \\
\hline $\mathrm{P} 1-\mathrm{C} 25$ & $1.893(4)$ & $\mathrm{P} 2-\mathrm{C} 61$ & $1.884(4)$ \\
\hline $\mathrm{O} 1-\mathrm{C} 18$ & $1.379(4)$ & $\mathrm{O} 3-\mathrm{C} 54$ & $1.380(5)$ \\
\hline $\mathrm{O} 1-\mathrm{C} 23$ & $1.446(4)$ & $\mathrm{O} 3-\mathrm{C} 59$ & $1.445(4)$ \\
\hline $\mathrm{O} 2-\mathrm{C} 22$ & $1.387(5)$ & $\mathrm{O} 4-\mathrm{C} 58$ & $1.390(5)$ \\
\hline $\mathrm{O} 2-\mathrm{C} 24$ & $1.443(5)$ & $\mathrm{O} 4-\mathrm{C} 60$ & $1.438(5)$ \\
\hline $\mathrm{C} 1-\mathrm{C} 6$ & $1.419(6)$ & $\mathrm{C} 37-\mathrm{C} 42$ & $1.416(7)$ \\
\hline $\mathrm{C} 1-\mathrm{C} 2$ & $1.443(5)$ & $\mathrm{C} 37-\mathrm{C} 38$ & $1.445(6)$ \\
\hline $\mathrm{C} 1-\mathrm{C} 7$ & $1.528(5)$ & $\mathrm{C} 37-\mathrm{C} 43$ & $1.532(7)$ \\
\hline $\mathrm{C} 2-\mathrm{C} 3$ & $1.405(6)$ & C $38-\mathrm{C} 39$ & $1.391(6)$ \\
\hline $\mathrm{C} 2-\mathrm{H} 2$ & 0.9500 & $\mathrm{C} 38-\mathrm{H} 38$ & 0.9500 \\
\hline $\mathrm{C} 3-\mathrm{C} 4$ & $1.435(7)$ & $\mathrm{C} 39-\mathrm{C} 40$ & $1.448(7)$ \\
\hline $\mathrm{C} 3-\mathrm{H} 3$ & 0.9500 & С $39-\mathrm{H} 39$ & 0.9500 \\
\hline $\mathrm{C} 4-\mathrm{C} 5$ & $1.435(6)$ & $\mathrm{C} 40-\mathrm{C} 41$ & $1.420(6)$ \\
\hline $\mathrm{C} 4-\mathrm{C} 10$ & $1.513(6)$ & $\mathrm{C} 40-\mathrm{C} 46$ & $1.510(7)$ \\
\hline $\mathrm{C} 5-\mathrm{C} 6$ & $1.440(5)$ & $\mathrm{C} 41-\mathrm{C} 42$ & $1.453(7)$ \\
\hline $\mathrm{C} 5-\mathrm{H} 5$ & 0.9500 & C41-H41 & 0.9500 \\
\hline C6- $-\mathrm{H} 6$ & 0.9500 & $\mathrm{C} 42-\mathrm{H} 42$ & 0.9500 \\
\hline C7-C9 & $1.509(7)$ & $\mathrm{C} 43-\mathrm{C} 45$ & $1.541(7)$ \\
\hline $\mathrm{C} 7-\mathrm{C} 8$ & $1.535(5)$ & $\mathrm{C} 43-\mathrm{C} 44$ & $1.544(6)$ \\
\hline $\mathrm{C} 7-\mathrm{H} 7$ & 1.0000 & $\mathrm{C} 43-\mathrm{H} 43$ & 1.0000 \\
\hline $\mathrm{C} 8-\mathrm{H} 8 \mathrm{~A}$ & 0.9800 & $\mathrm{C} 44-\mathrm{H} 44 \mathrm{~A}$ & 0.9800 \\
\hline $\mathrm{C} 8-\mathrm{H} 8 \mathrm{~B}$ & 0.9800 & $\mathrm{C} 44-\mathrm{H} 44 \mathrm{~B}$ & 0.9800 \\
\hline $\mathrm{C} 8-\mathrm{H} 8 \mathrm{C}$ & 0.9800 & $\mathrm{C} 44-\mathrm{H} 44 \mathrm{C}$ & 0.9800 \\
\hline C9- $\mathrm{H} 9 \mathrm{~A}$ & 0.9800 & $\mathrm{C} 45-\mathrm{H} 45 \mathrm{~A}$ & 0.9800 \\
\hline C9-H9B & 0.9800 & $\mathrm{C} 45-\mathrm{H} 45 \mathrm{~B}$ & 0.9800 \\
\hline C9-H9C & 0.9800 & $\mathrm{C} 45-\mathrm{H} 45 \mathrm{C}$ & 0.9800 \\
\hline $\mathrm{C} 10-\mathrm{H} 10 \mathrm{~A}$ & 0.9800 & $\mathrm{C} 46-\mathrm{H} 46 \mathrm{~A}$ & 0.9800 \\
\hline $\mathrm{C} 10-\mathrm{H} 10 \mathrm{~B}$ & 0.9800 & $\mathrm{C} 46-\mathrm{H} 46 \mathrm{~B}$ & 0.9800 \\
\hline $\mathrm{C} 10-\mathrm{H} 10 \mathrm{C}$ & 0.9800 & $\mathrm{C} 46-\mathrm{H} 46 \mathrm{C}$ & 0.9800 \\
\hline $\mathrm{C} 11-\mathrm{C} 12$ & $1.419(5)$ & $\mathrm{C} 47-\mathrm{C} 52$ & $1.418(6)$ \\
\hline $\mathrm{C} 11-\mathrm{C} 16$ & $1.428(5)$ & $\mathrm{C} 47-\mathrm{C} 48$ & $1.437(5)$ \\
\hline $\mathrm{C} 12-\mathrm{C} 13$ & $1.420(6)$ & $\mathrm{C} 48-\mathrm{C} 49$ & $1.411(6)$ \\
\hline $\mathrm{C} 12-\mathrm{C} 17$ & $1.516(5)$ & $\mathrm{C} 48-\mathrm{C} 53$ & $1.513(6)$ \\
\hline $\mathrm{C} 13-\mathrm{C} 14$ & $1.394(6)$ & $\mathrm{C} 49-\mathrm{C} 50$ & $1.394(6)$ \\
\hline $\mathrm{C} 13-\mathrm{H} 13$ & 0.9500 & $\mathrm{C} 49-\mathrm{H} 49$ & 0.9500 \\
\hline
\end{tabular}




\begin{tabular}{|c|c|c|c|}
\hline $\mathrm{C} 14-\mathrm{C} 15$ & $1.400(6)$ & $\mathrm{C} 50-\mathrm{C} 51$ & $1.400(6)$ \\
\hline C14-H14 & 0.9500 & $\mathrm{C} 50-\mathrm{H} 50$ & 0.9500 \\
\hline $\mathrm{C} 15-\mathrm{C} 16$ & $1.393(6)$ & $\mathrm{C} 51-\mathrm{C} 52$ & $1.397(6)$ \\
\hline $\mathrm{C} 15-\mathrm{H} 15$ & 0.9500 & C51-H51 & 0.9500 \\
\hline $\mathrm{C} 16-\mathrm{H} 16$ & 0.9500 & C52-H52 & 0.9500 \\
\hline $\mathrm{C} 17-\mathrm{C} 18$ & $1.411(5)$ & $\mathrm{C} 53-\mathrm{C} 54$ & $1.414(6)$ \\
\hline $\mathrm{C} 17-\mathrm{C} 22$ & $1.417(5)$ & $\mathrm{C} 53-\mathrm{C} 58$ & $1.416(6)$ \\
\hline $\mathrm{C} 18-\mathrm{C} 19$ & $1.414(5)$ & $\mathrm{C} 54-\mathrm{C} 55$ & $1.417(5)$ \\
\hline $\mathrm{C} 19-\mathrm{C} 20$ & $1.407(6)$ & $\mathrm{C} 55-\mathrm{C} 56$ & $1.407(6)$ \\
\hline C19-H19 & 0.9500 & $\mathrm{C} 55-\mathrm{H} 55$ & 0.9500 \\
\hline $\mathrm{C} 20-\mathrm{C} 21$ & $1.394(6)$ & $\mathrm{C} 56-\mathrm{C} 57$ & $1.392(7)$ \\
\hline $\mathrm{C} 20-\mathrm{H} 20$ & 0.9500 & C56-H56 & 0.9500 \\
\hline $\mathrm{C} 21-\mathrm{C} 22$ & $1.408(5)$ & $\mathrm{C} 57-\mathrm{C} 58$ & $1.401(6)$ \\
\hline $\mathrm{C} 21-\mathrm{H} 21$ & 0.9500 & C57-H57 & 0.9500 \\
\hline $\mathrm{C} 23-\mathrm{H} 23 \mathrm{~A}$ & 0.9800 & C59-H59A & 0.9800 \\
\hline $\mathrm{C} 23-\mathrm{H} 23 \mathrm{~B}$ & 0.9800 & $\mathrm{C} 59-\mathrm{H} 59 \mathrm{~B}$ & 0.9800 \\
\hline $\mathrm{C} 23-\mathrm{H} 23 \mathrm{C}$ & 0.9800 & C59-H59C & 0.9800 \\
\hline $\mathrm{C} 24-\mathrm{H} 24 \mathrm{~A}$ & 0.9800 & $\mathrm{C} 60-\mathrm{H} 60 \mathrm{~A}$ & 0.9800 \\
\hline $\mathrm{C} 24-\mathrm{H} 24 \mathrm{~B}$ & 0.9800 & C60-H60B & 0.9800 \\
\hline $\mathrm{C} 24-\mathrm{H} 24 \mathrm{C}$ & 0.9800 & $\mathrm{C} 60-\mathrm{H} 60 \mathrm{C}$ & 0.9800 \\
\hline $\mathrm{C} 25-\mathrm{C} 26$ & $1.546(5)$ & C61-C62 & $1.550(6)$ \\
\hline $\mathrm{C} 25-\mathrm{C} 30$ & $1.552(5)$ & C61-C66 & $1.558(5)$ \\
\hline $\mathrm{C} 25-\mathrm{H} 25$ & 1.0000 & C61-H61 & 1.0000 \\
\hline $\mathrm{C} 26-\mathrm{C} 27$ & $1.540(5)$ & C62-C63 & $1.537(6)$ \\
\hline $\mathrm{C} 26-\mathrm{H} 26 \mathrm{~A}$ & 0.9900 & $\mathrm{C} 62-\mathrm{H} 62 \mathrm{~A}$ & 0.9900 \\
\hline $\mathrm{C} 26-\mathrm{H} 26 \mathrm{~B}$ & 0.9900 & C62-H62B & 0.9900 \\
\hline $\mathrm{C} 27-\mathrm{C} 28$ & $1.549(6)$ & C63-C64 & $1.540(6)$ \\
\hline $\mathrm{C} 27-\mathrm{H} 27 \mathrm{~A}$ & 0.9900 & $\mathrm{C} 63-\mathrm{H} 63 \mathrm{~A}$ & 0.9900 \\
\hline $\mathrm{C} 27-\mathrm{H} 27 \mathrm{~B}$ & 0.9900 & C63-H63B & 0.9900 \\
\hline $\mathrm{C} 28-\mathrm{C} 29$ & $1.542(6)$ & C64-C65 & $1.538(6)$ \\
\hline $\mathrm{C} 28-\mathrm{H} 28 \mathrm{~A}$ & 0.9900 & $\mathrm{C} 64-\mathrm{H} 64 \mathrm{~A}$ & 0.9900 \\
\hline $\mathrm{C} 28-\mathrm{H} 28 \mathrm{~B}$ & 0.9900 & C64-H64B & 0.9900 \\
\hline $\mathrm{C} 29-\mathrm{C} 30$ & $1.537(5)$ & C65-C66 & $1.551(6)$ \\
\hline $\mathrm{C} 29-\mathrm{H} 29 \mathrm{~A}$ & 0.9900 & C65-H65A & 0.9900 \\
\hline $\mathrm{C} 29-\mathrm{H} 29 \mathrm{~B}$ & 0.9900 & C65-H65B & 0.9900 \\
\hline $\mathrm{C} 30-\mathrm{H} 30 \mathrm{~A}$ & 0.9900 & C66-H66A & 0.9900 \\
\hline $\mathrm{C} 30-\mathrm{H} 30 \mathrm{~B}$ & 0.9900 & C66-H66B & 0.9900 \\
\hline C31-C32 & $1.541(5)$ & C67-C68 & $1.546(6)$ \\
\hline $\mathrm{C} 31-\mathrm{C} 36$ & $1.558(5)$ & $\mathrm{C} 67-\mathrm{C} 72$ & $1.553(6)$ \\
\hline $\mathrm{C} 31-\mathrm{H} 31$ & 1.0000 & C67-H67 & 1.0000 \\
\hline $\mathrm{C} 32-\mathrm{C} 33$ & $1.544(5)$ & C68-C69 & $1.551(6)$ \\
\hline $\mathrm{C} 32-\mathrm{H} 32 \mathrm{~A}$ & 0.9900 & C68-H68A & 0.9900 \\
\hline $\mathrm{C} 32-\mathrm{H} 32 \mathrm{~B}$ & 0.9900 & C68-H68B & 0.9900 \\
\hline C33-C34 & $1.538(6)$ & $\mathrm{C} 69-\mathrm{C} 70$ & $1.537(7)$ \\
\hline $\mathrm{C} 33-\mathrm{H} 33 \mathrm{~A}$ & 0.9900 & $\mathrm{C} 69-\mathrm{H} 69 \mathrm{~A}$ & 0.9900 \\
\hline C33-H33B & 0.9900 & C69-H69B & 0.9900 \\
\hline C34-C35 & $1.538(6)$ & $\mathrm{C} 70-\mathrm{C} 71$ & $1.527(8)$ \\
\hline $\mathrm{C} 34-\mathrm{H} 34 \mathrm{~A}$ & 0.9900 & $\mathrm{C} 70-\mathrm{H} 70 \mathrm{~A}$ & 0.9900 \\
\hline
\end{tabular}




\begin{tabular}{|c|c|c|c|}
\hline C $34-\mathrm{H} 34 \mathrm{~B}$ & 0.9900 & $\mathrm{C} 70-\mathrm{H} 70 \mathrm{~B}$ & 0.9900 \\
\hline $\mathrm{C} 35-\mathrm{C} 36$ & $1.539(5)$ & $\mathrm{C} 71-\mathrm{C} 72$ & $1.535(6)$ \\
\hline $\mathrm{C} 35-\mathrm{H} 35 \mathrm{~A}$ & 0.9900 & C71-H71A & 0.9900 \\
\hline C $35-\mathrm{H} 35 \mathrm{~B}$ & 0.9900 & C71-H71B & 0.9900 \\
\hline $\mathrm{C} 36-\mathrm{H} 36 \mathrm{~A}$ & 0.9900 & $\mathrm{C} 72-\mathrm{H} 72 \mathrm{~A}$ & 0.9900 \\
\hline $\mathrm{C} 36-\mathrm{H} 36 \mathrm{~B}$ & 0.9900 & $\mathrm{C} 72-\mathrm{H} 72 \mathrm{~B}$ & 0.9900 \\
\hline $\mathrm{C} 6-\mathrm{Ru} 1-\mathrm{C} 5$ & $37.92(14)$ & $\mathrm{C} 41-\mathrm{Ru} 2-\mathrm{C} 42$ & $38.05(18)$ \\
\hline $\mathrm{C} 6-\mathrm{Ru} 1-\mathrm{C} 4$ & $67.73(14)$ & $\mathrm{C} 41-\mathrm{Ru} 2-\mathrm{C} 40$ & $37.03(17)$ \\
\hline $\mathrm{C} 5-\mathrm{Ru} 1-\mathrm{C} 4$ & $37.40(15)$ & $\mathrm{C} 42-\mathrm{Ru} 2-\mathrm{C} 40$ & $67.57(16)$ \\
\hline $\mathrm{C} 6-\mathrm{Ru} 1-\mathrm{C} 2$ & $66.40(14)$ & $\mathrm{C} 41-\mathrm{Ru} 2-\mathrm{C} 38$ & $78.30(17)$ \\
\hline $\mathrm{C} 5-\mathrm{Ru} 1-\mathrm{C} 2$ & $78.81(15)$ & $\mathrm{C} 42-\mathrm{Ru} 2-\mathrm{C} 38$ & $65.82(15)$ \\
\hline $\mathrm{C} 4-\mathrm{Ru} 1-\mathrm{C} 2$ & $66.52(16)$ & $\mathrm{C} 40-\mathrm{Ru} 2-\mathrm{C} 38$ & 66.44 (17) \\
\hline $\mathrm{C} 6-\mathrm{Ru} 1-\mathrm{C} 1$ & $36.97(15)$ & $\mathrm{C} 41-\mathrm{Ru} 2-\mathrm{C} 39$ & $66.45(17)$ \\
\hline $\mathrm{C} 5-\mathrm{Ru} 1-\mathrm{C} 1$ & $67.67(14)$ & $\mathrm{C} 42-\mathrm{Ru} 2-\mathrm{C} 39$ & $78.11(15)$ \\
\hline $\mathrm{C} 4-\mathrm{Ru} 1-\mathrm{C} 1$ & 79.89 (14) & $\mathrm{C} 40-\mathrm{Ru} 2-\mathrm{C} 39$ & $37.45(18)$ \\
\hline $\mathrm{C} 2-\mathrm{Ru} 1-\mathrm{C} 1$ & $37.20(13)$ & $\mathrm{C} 38-\mathrm{Ru} 2-\mathrm{C} 39$ & $35.77(16)$ \\
\hline $\mathrm{C} 6-\mathrm{Ru} 1-\mathrm{C} 3$ & $78.43(14)$ & $\mathrm{C} 41-\mathrm{Ru} 2-\mathrm{C} 37$ & $67.52(18)$ \\
\hline $\mathrm{C} 5-\mathrm{Ru} 1-\mathrm{C} 3$ & $66.58(15)$ & $\mathrm{C} 42-\mathrm{Ru} 2-\mathrm{C} 37$ & $36.61(17)$ \\
\hline $\mathrm{C} 4-\mathrm{Ru} 1-\mathrm{C} 3$ & $37.03(17)$ & $\mathrm{C} 40-\mathrm{Ru} 2-\mathrm{C} 37$ & $79.91(17)$ \\
\hline $\mathrm{C} 2-\mathrm{Ru} 1-\mathrm{C} 3$ & $36.15(15)$ & $\mathrm{C} 38-\mathrm{Ru} 2-\mathrm{C} 37$ & $37.12(14)$ \\
\hline $\mathrm{C} 1-\mathrm{Ru} 1-\mathrm{C} 3$ & $66.44(14)$ & $\mathrm{C} 39-\mathrm{Ru} 2-\mathrm{C} 37$ & $66.26(15)$ \\
\hline $\mathrm{C} 6-\mathrm{Ru} 1-\mathrm{P} 1$ & $93.14(10)$ & $\mathrm{C} 41-\mathrm{Ru} 2-\mathrm{P} 2$ & $96.33(13)$ \\
\hline $\mathrm{C} 5-\mathrm{Ru} 1-\mathrm{P} 1$ & $96.05(11)$ & $\mathrm{C} 42-\mathrm{Ru} 2-\mathrm{P} 2$ & $94.49(11)$ \\
\hline $\mathrm{C} 4-\mathrm{Ru} 1-\mathrm{P} 1$ & $123.63(13)$ & $\mathrm{C} 40-\mathrm{Ru} 2-\mathrm{P} 2$ & $122.70(14)$ \\
\hline $\mathrm{C} 2-\mathrm{Ru} 1-\mathrm{P} 1$ & $152.93(10)$ & $\mathrm{C} 38-\mathrm{Ru} 2-\mathrm{P} 2$ & $154.49(11)$ \\
\hline $\mathrm{C} 1-\mathrm{Ru} 1-\mathrm{P} 1$ & $116.23(10)$ & $\mathrm{C} 39-\mathrm{Ru} 2-\mathrm{P} 2$ & $160.14(12)$ \\
\hline $\mathrm{C} 3-\mathrm{Ru} 1-\mathrm{P} 1$ & $160.65(13)$ & $\mathrm{C} 37-\mathrm{Ru} 2-\mathrm{P} 2$ & $117.74(11)$ \\
\hline $\mathrm{C} 6-\mathrm{Ru} 1-\mathrm{Cl1}$ & $147.88(11)$ & $\mathrm{C} 41-\mathrm{Ru} 2-\mathrm{Cl} 3$ & $162.00(14)$ \\
\hline $\mathrm{C} 5-\mathrm{Ru} 1-\mathrm{Cl1}$ & $110.06(10)$ & $\mathrm{C} 42-\mathrm{Ru} 2-\mathrm{Cl} 3$ & $124.32(13)$ \\
\hline $\mathrm{C} 4-\mathrm{Ru} 1-\mathrm{Cl} 1$ & $85.41(10)$ & $\mathrm{C} 40-\mathrm{Ru} 2-\mathrm{Cl} 3$ & $147.92(13)$ \\
\hline $\mathrm{C} 2-\mathrm{Ru} 1-\mathrm{Cl} 1$ & $119.60(10)$ & $\mathrm{C} 38-\mathrm{Ru} 2-\mathrm{Cl} 3$ & 90.39 (11) \\
\hline $\mathrm{C} 1-\mathrm{Ru} 1-\mathrm{C} 11$ & $156.45(10)$ & $\mathrm{C} 39-\mathrm{Ru} 2-\mathrm{Cl} 3$ & $111.60(12)$ \\
\hline $\mathrm{C} 3-\mathrm{Ru} 1-\mathrm{Cl} 1$ & $90.82(10)$ & $\mathrm{C} 37-\mathrm{Ru} 2-\mathrm{Cl} 3$ & $95.02(12)$ \\
\hline $\mathrm{P} 1-\mathrm{Ru} 1-\mathrm{C} 11$ & $87.23(4)$ & $\mathrm{P} 2-\mathrm{Ru} 2-\mathrm{Cl} 3$ & $87.86(4)$ \\
\hline $\mathrm{C} 6-\mathrm{Ru} 1-\mathrm{Cl} 2$ & $124.08(11)$ & $\mathrm{C} 41-\mathrm{Ru} 2-\mathrm{Cl} 4$ & $111.36(14)$ \\
\hline $\mathrm{C} 5-\mathrm{Ru} 1-\mathrm{Cl} 2$ & $161.65(10)$ & $\mathrm{C} 42-\mathrm{Ru} 2-\mathrm{Cl} 4$ & $149.39(13)$ \\
\hline $\mathrm{C} 4-\mathrm{Ru} 1-\mathrm{Cl} 2$ & $147.67(13)$ & $\mathrm{C} 40-\mathrm{Ru} 2-\mathrm{Cl} 4$ & $85.79(13)$ \\
\hline $\mathrm{C} 2-\mathrm{Ru} 1-\mathrm{Cl} 2$ & $89.81(11)$ & $\mathrm{C} 38-\mathrm{Ru} 2-\mathrm{Cl} 4$ & $118.01(10)$ \\
\hline $\mathrm{C} 1-\mathrm{Ru} 1-\mathrm{Cl} 2$ & $94.58(10)$ & $\mathrm{C} 39-\mathrm{Ru} 2-\mathrm{Cl} 4$ & 89.98 (12) \\
\hline $\mathrm{C} 3-\mathrm{Ru} 1-\mathrm{Cl} 2$ & $111.66(12)$ & $\mathrm{C} 37-\mathrm{Ru} 2-\mathrm{Cl} 4$ & $154.97(11)$ \\
\hline $\mathrm{P} 1-\mathrm{Ru} 1-\mathrm{Cl} 2$ & $87.53(4)$ & $\mathrm{P} 2-\mathrm{Ru} 2-\mathrm{Cl} 4$ & $87.28(4)$ \\
\hline $\mathrm{C} 11-\mathrm{Ru} 1-\mathrm{Cl} 2$ & $88.04(4)$ & $\mathrm{Cl} 3-\mathrm{Ru} 2-\mathrm{Cl} 4$ & $86.26(4)$ \\
\hline $\mathrm{C} 11-\mathrm{P} 1-\mathrm{C} 31$ & $108.60(17)$ & $\mathrm{C} 47-\mathrm{P} 2-\mathrm{C} 67$ & $108.53(18)$ \\
\hline $\mathrm{C} 11-\mathrm{P} 1-\mathrm{C} 25$ & $96.86(17)$ & $\mathrm{C} 47-\mathrm{P} 2-\mathrm{C} 61$ & 97.24 (19) \\
\hline $\mathrm{C} 31-\mathrm{P} 1-\mathrm{C} 25$ & $106.60(16)$ & $\mathrm{C} 67-\mathrm{P} 2-\mathrm{C} 61$ & $106.12(18)$ \\
\hline $\mathrm{C} 11-\mathrm{P} 1-\mathrm{Ru} 1$ & $108.18(11)$ & $\mathrm{C} 47-\mathrm{P} 2-\mathrm{Ru} 2$ & $108.16(12)$ \\
\hline $\mathrm{C} 31-\mathrm{P} 1-\mathrm{Ru} 1$ & $119.13(13)$ & $\mathrm{C} 67-\mathrm{P} 2-\mathrm{Ru} 2$ & $117.37(14)$ \\
\hline
\end{tabular}




\begin{tabular}{|c|c|c|c|}
\hline $\mathrm{C} 25-\mathrm{P} 1-\mathrm{Ru} 1$ & $115.06(11)$ & $\mathrm{C} 61-\mathrm{P} 2-\mathrm{Ru} 2$ & $117.35(12)$ \\
\hline $\mathrm{C} 18-\mathrm{O} 1-\mathrm{C} 23$ & $116.7(3)$ & $\mathrm{C} 54-\mathrm{O} 3-\mathrm{C} 59$ & $117.3(3)$ \\
\hline $\mathrm{C} 22-\mathrm{O} 2-\mathrm{C} 24$ & $117.6(3)$ & $\mathrm{C} 58-\mathrm{O} 4-\mathrm{C} 60$ & $117.9(4)$ \\
\hline $\mathrm{C} 6-\mathrm{C} 1-\mathrm{C} 2$ & $117.3(3)$ & $\mathrm{C} 42-\mathrm{C} 37-\mathrm{C} 38$ & $117.2(4)$ \\
\hline $\mathrm{C} 6-\mathrm{C} 1-\mathrm{C} 7$ & $120.0(3)$ & $\mathrm{C} 42-\mathrm{C} 37-\mathrm{C} 43$ & $121.7(4)$ \\
\hline $\mathrm{C} 2-\mathrm{C} 1-\mathrm{C} 7$ & $122.2(4)$ & $\mathrm{C} 38-\mathrm{C} 37-\mathrm{C} 43$ & $120.3(4)$ \\
\hline $\mathrm{C} 6-\mathrm{C} 1-\mathrm{Ru} 1$ & 69.20 (19) & $\mathrm{C} 42-\mathrm{C} 37-\mathrm{Ru} 2$ & $69.9(2)$ \\
\hline $\mathrm{C} 2-\mathrm{C} 1-\mathrm{Ru} 1$ & $71.12(18)$ & $\mathrm{C} 38-\mathrm{C} 37-\mathrm{Ru} 2$ & $71.0(2)$ \\
\hline $\mathrm{C} 7-\mathrm{C} 1-\mathrm{Ru} 1$ & $137.1(2)$ & $\mathrm{C} 43-\mathrm{C} 37-\mathrm{Ru} 2$ & $138.6(3)$ \\
\hline $\mathrm{C} 3-\mathrm{C} 2-\mathrm{C} 1$ & $121.5(4)$ & $\mathrm{C} 39-\mathrm{C} 38-\mathrm{C} 37$ & $122.1(4)$ \\
\hline $\mathrm{C} 3-\mathrm{C} 2-\mathrm{Ru} 1$ & $72.4(2)$ & $\mathrm{C} 39-\mathrm{C} 38-\mathrm{Ru} 2$ & $72.2(2)$ \\
\hline $\mathrm{C} 1-\mathrm{C} 2-\mathrm{Ru} 1$ & 71.69 (19) & $\mathrm{C} 37-\mathrm{C} 38-\mathrm{Ru} 2$ & $71.9(2)$ \\
\hline $\mathrm{C} 3-\mathrm{C} 2-\mathrm{H} 2$ & 119.2 & $\mathrm{C} 39-\mathrm{C} 38-\mathrm{H} 38$ & 118.9 \\
\hline $\mathrm{C} 1-\mathrm{C} 2-\mathrm{H} 2$ & 119.2 & $\mathrm{C} 37-\mathrm{C} 38-\mathrm{H} 38$ & 118.9 \\
\hline $\mathrm{Ru} 1-\mathrm{C} 2-\mathrm{H} 2$ & 129.1 & $\mathrm{Ru} 2-\mathrm{C} 38-\mathrm{H} 38$ & 129.6 \\
\hline $\mathrm{C} 2-\mathrm{C} 3-\mathrm{C} 4$ & $120.9(4)$ & $\mathrm{C} 38-\mathrm{C} 39-\mathrm{C} 40$ & $120.9(4)$ \\
\hline $\mathrm{C} 2-\mathrm{C} 3-\mathrm{Ru} 1$ & $71.5(2)$ & $\mathrm{C} 38-\mathrm{C} 39-\mathrm{Ru} 2$ & $72.0(2)$ \\
\hline $\mathrm{C} 4-\mathrm{C} 3-\mathrm{Ru} 1$ & $70.62(19)$ & $\mathrm{C} 40-\mathrm{C} 39-\mathrm{Ru} 2$ & $70.5(2)$ \\
\hline $\mathrm{C} 2-\mathrm{C} 3-\mathrm{H} 3$ & 119.5 & C $38-\mathrm{C} 39-\mathrm{H} 39$ & 119.6 \\
\hline $\mathrm{C} 4-\mathrm{C} 3-\mathrm{H} 3$ & 119.5 & $\mathrm{C} 40-\mathrm{C} 39-\mathrm{H} 39$ & 119.6 \\
\hline $\mathrm{Ru} 1-\mathrm{C} 3-\mathrm{H} 3$ & 131.3 & $\mathrm{Ru} 2-\mathrm{C} 39-\mathrm{H} 39$ & 130.6 \\
\hline $\mathrm{C} 3-\mathrm{C} 4-\mathrm{C} 5$ & $118.7(4)$ & $\mathrm{C} 41-\mathrm{C} 40-\mathrm{C} 39$ & $118.3(4)$ \\
\hline $\mathrm{C} 3-\mathrm{C} 4-\mathrm{C} 10$ & $120.8(4)$ & $\mathrm{C} 41-\mathrm{C} 40-\mathrm{C} 46$ & $122.0(5)$ \\
\hline $\mathrm{C} 5-\mathrm{C} 4-\mathrm{C} 10$ & $120.5(4)$ & $\mathrm{C} 39-\mathrm{C} 40-\mathrm{C} 46$ & $119.7(4)$ \\
\hline $\mathrm{C} 3-\mathrm{C} 4-\mathrm{Ru} 1$ & $72.3(2)$ & $\mathrm{C} 41-\mathrm{C} 40-\mathrm{Ru} 2$ & $70.9(2)$ \\
\hline $\mathrm{C} 5-\mathrm{C} 4-\mathrm{Ru} 1$ & $70.53(19)$ & $\mathrm{C} 39-\mathrm{C} 40-\mathrm{Ru} 2$ & $72.1(2)$ \\
\hline $\mathrm{C} 10-\mathrm{C} 4-\mathrm{Ru} 1$ & $129.9(3)$ & $\mathrm{C} 46-\mathrm{C} 40-\mathrm{Ru} 2$ & $128.4(3)$ \\
\hline $\mathrm{C} 4-\mathrm{C} 5-\mathrm{C} 6$ & $119.3(4)$ & $\mathrm{C} 40-\mathrm{C} 41-\mathrm{C} 42$ & $120.0(4)$ \\
\hline $\mathrm{C} 4-\mathrm{C} 5-\mathrm{Ru} 1$ & $72.1(2)$ & $\mathrm{C} 40-\mathrm{C} 41-\mathrm{Ru} 2$ & $72.1(2)$ \\
\hline $\mathrm{C} 6-\mathrm{C} 5-\mathrm{Ru} 1$ & $70.21(19)$ & $\mathrm{C} 42-\mathrm{C} 41-\mathrm{Ru} 2$ & $71.0(2)$ \\
\hline $\mathrm{C} 4-\mathrm{C} 5-\mathrm{H} 5$ & 120.3 & $\mathrm{C} 40-\mathrm{C} 41-\mathrm{H} 41$ & 120.0 \\
\hline $\mathrm{C} 6-\mathrm{C} 5-\mathrm{H} 5$ & 120.3 & $\mathrm{C} 42-\mathrm{C} 41-\mathrm{H} 41$ & 120.0 \\
\hline $\mathrm{Ru} 1-\mathrm{C} 5-\mathrm{H} 5$ & 129.8 & $\mathrm{Ru} 2-\mathrm{C} 41-\mathrm{H} 41$ & 129.2 \\
\hline $\mathrm{C} 1-\mathrm{C} 6-\mathrm{C} 5$ & $122.1(3)$ & $\mathrm{C} 37-\mathrm{C} 42-\mathrm{C} 41$ & $121.5(4)$ \\
\hline $\mathrm{C} 1-\mathrm{C} 6-\mathrm{Ru} 1$ & 73.83 (19) & $\mathrm{C} 37-\mathrm{C} 42-\mathrm{Ru} 2$ & $73.5(2)$ \\
\hline $\mathrm{C} 5-\mathrm{C} 6-\mathrm{Ru} 1$ & 71.87 (19) & $\mathrm{C} 41-\mathrm{C} 42-\mathrm{Ru} 2$ & $70.9(2)$ \\
\hline $\mathrm{C} 1-\mathrm{C} 6-\mathrm{H} 6$ & 119.0 & $\mathrm{C} 37-\mathrm{C} 42-\mathrm{H} 42$ & 119.2 \\
\hline $\mathrm{C} 5-\mathrm{C} 6-\mathrm{H} 6$ & 119.0 & $\mathrm{C} 41-\mathrm{C} 42-\mathrm{H} 42$ & 119.2 \\
\hline $\mathrm{Ru} 1-\mathrm{C} 6-\mathrm{H} 6$ & 127.5 & $\mathrm{Ru} 2-\mathrm{C} 42-\mathrm{H} 42$ & 128.8 \\
\hline $\mathrm{C} 9-\mathrm{C} 7-\mathrm{C} 1$ & $116.4(4)$ & $\mathrm{C} 37-\mathrm{C} 43-\mathrm{C} 45$ & $114.9(4)$ \\
\hline $\mathrm{C} 9-\mathrm{C} 7-\mathrm{C} 8$ & $110.5(4)$ & $\mathrm{C} 37-\mathrm{C} 43-\mathrm{C} 44$ & $107.7(4)$ \\
\hline $\mathrm{C} 1-\mathrm{C} 7-\mathrm{C} 8$ & $106.9(3)$ & $\mathrm{C} 45-\mathrm{C} 43-\mathrm{C} 44$ & $109.9(4)$ \\
\hline $\mathrm{C} 9-\mathrm{C} 7-\mathrm{H} 7$ & 107.6 & $\mathrm{C} 37-\mathrm{C} 43-\mathrm{H} 43$ & 108.0 \\
\hline $\mathrm{C} 1-\mathrm{C} 7-\mathrm{H} 7$ & 107.6 & $\mathrm{C} 45-\mathrm{C} 43-\mathrm{H} 43$ & 108.0 \\
\hline $\mathrm{C} 8-\mathrm{C} 7-\mathrm{H} 7$ & 107.6 & $\mathrm{C} 44-\mathrm{C} 43-\mathrm{H} 43$ & 108.0 \\
\hline $\mathrm{C} 7-\mathrm{C} 8-\mathrm{H} 8 \mathrm{~A}$ & 109.5 & $\mathrm{C} 43-\mathrm{C} 44-\mathrm{H} 44 \mathrm{~A}$ & 109.5 \\
\hline $\mathrm{C} 7-\mathrm{C} 8-\mathrm{H} 8 \mathrm{~B}$ & 109.5 & $\mathrm{C} 43-\mathrm{C} 44-\mathrm{H} 44 \mathrm{~B}$ & 109.5 \\
\hline $\mathrm{H} 8 \mathrm{~A}-\mathrm{C} 8-\mathrm{H} 8 \mathrm{~B}$ & 109.5 & $\mathrm{H} 44 \mathrm{~A}-\mathrm{C} 44-\mathrm{H} 44 \mathrm{~B}$ & 109.5 \\
\hline
\end{tabular}


$\mathrm{C} 7-\mathrm{C} 8-\mathrm{H} 8 \mathrm{C}$

$\mathrm{H} 8 \mathrm{~A}-\mathrm{C} 8-\mathrm{H} 8 \mathrm{C}$

$\mathrm{H} 8 \mathrm{~B}-\mathrm{C} 8-\mathrm{H} 8 \mathrm{C}$

$\mathrm{C} 7-\mathrm{C} 9-\mathrm{H} 9 \mathrm{~A}$

$\mathrm{C} 7-\mathrm{C} 9-\mathrm{H} 9 \mathrm{~B}$

$\mathrm{H} 9 \mathrm{~A}-\mathrm{C} 9-\mathrm{H} 9 \mathrm{~B}$

$\mathrm{C} 7-\mathrm{C} 9-\mathrm{H} 9 \mathrm{C}$

$\mathrm{H} 9 \mathrm{~A}-\mathrm{C} 9-\mathrm{H} 9 \mathrm{C}$

$\mathrm{H} 9 \mathrm{~B}-\mathrm{C} 9-\mathrm{H} 9 \mathrm{C}$

C4- $\mathrm{C} 10-\mathrm{H} 10 \mathrm{~A}$

C4-C10-H10B

$\mathrm{H} 10 \mathrm{~A}-\mathrm{C} 10-\mathrm{H} 10 \mathrm{~B}$

C4- $\mathrm{C} 10-\mathrm{H} 10 \mathrm{C}$

$\mathrm{H} 10 \mathrm{~A}-\mathrm{C} 10-\mathrm{H} 10 \mathrm{C}$

H10B-C10-H10C

$\mathrm{C} 12-\mathrm{C} 11-\mathrm{C} 16$

$\mathrm{C} 12-\mathrm{C} 11-\mathrm{P} 1$

$\mathrm{C} 16-\mathrm{C} 11-\mathrm{P} 1$

$\mathrm{C} 11-\mathrm{C} 12-\mathrm{C} 13$

$\mathrm{C} 11-\mathrm{C} 12-\mathrm{C} 17$

$\mathrm{C} 13-\mathrm{C} 12-\mathrm{C} 17$

$\mathrm{C} 14-\mathrm{C} 13-\mathrm{C} 12$

$\mathrm{C} 14-\mathrm{C} 13-\mathrm{H} 13$

C12-C13-H13

$\mathrm{C} 13-\mathrm{C} 14-\mathrm{C} 15$

C13-C14-H14

C15-C14-H14

$\mathrm{C} 16-\mathrm{C} 15-\mathrm{C} 14$

C16- $15-\mathrm{H} 15$

C14-C15-H15

C15-C16-C11

C15-C16-H16

$\mathrm{C} 11-\mathrm{C} 16-\mathrm{H} 16$

$\mathrm{C} 18-\mathrm{C} 17-\mathrm{C} 22$

$\mathrm{C} 18-\mathrm{C} 17-\mathrm{C} 12$

$\mathrm{C} 22-\mathrm{C} 17-\mathrm{C} 12$

$\mathrm{O} 1-\mathrm{C} 18-\mathrm{C} 17$

$\mathrm{O} 1-\mathrm{C} 18-\mathrm{C} 19$

C17-C18-C19

$\mathrm{C} 20-\mathrm{C} 19-\mathrm{C} 18$

$\mathrm{C} 20-\mathrm{C} 19-\mathrm{H} 19$

C18-C19-H19

$\mathrm{C} 21-\mathrm{C} 20-\mathrm{C} 19$

$\mathrm{C} 21-\mathrm{C} 20-\mathrm{H} 20$

$\mathrm{C} 19-\mathrm{C} 20-\mathrm{H} 20$

$\mathrm{C} 20-\mathrm{C} 21-\mathrm{C} 22$

$\mathrm{C} 20-\mathrm{C} 21-\mathrm{H} 21$

$\mathrm{C} 22-\mathrm{C} 21-\mathrm{H} 21$
109.5

109.5

109.5

109.5

109.5

109.5

109.5

109.5

109.5

109.5

109.5

109.5

109.5

109.5

109.5

$118.3(3)$

130.7 (3)

$110.9(3)$

117.7 (3)

$128.2(4)$

$114.1(3)$

$123.1(4)$

118.5

118.5

119.0 (4)

120.5

120.5

119.3 (4)

120.4

120.4

122.4 (4)

118.8

118.8

$117.8(3)$

$121.7(3)$

$119.9(3)$

$115.1(3)$

$123.3(3)$

$121.6(3)$

118.5 (4)

120.8

120.8

121.5 (4)

119.2

119.2

$119.1(4)$

120.5

120.5
$\mathrm{C} 43-\mathrm{C} 44-\mathrm{H} 44 \mathrm{C}$

$\mathrm{H} 44 \mathrm{~A}-\mathrm{C} 44-\mathrm{H} 44 \mathrm{C}$

$\mathrm{H} 44 \mathrm{~B}-\mathrm{C} 44-\mathrm{H} 44 \mathrm{C}$

$\mathrm{C} 43-\mathrm{C} 45-\mathrm{H} 45 \mathrm{~A}$

C $43-\mathrm{C} 45-\mathrm{H} 45 \mathrm{~B}$

$\mathrm{H} 45 \mathrm{~A}-\mathrm{C} 45-\mathrm{H} 45 \mathrm{~B}$

$\mathrm{C} 43-\mathrm{C} 45-\mathrm{H} 45 \mathrm{C}$

$\mathrm{H} 45 \mathrm{~A}-\mathrm{C} 45-\mathrm{H} 45 \mathrm{C}$

$\mathrm{H} 45 \mathrm{~B}-\mathrm{C} 45-\mathrm{H} 45 \mathrm{C}$

$\mathrm{C} 40-\mathrm{C} 46-\mathrm{H} 46 \mathrm{~A}$

$\mathrm{C} 40-\mathrm{C} 46-\mathrm{H} 46 \mathrm{~B}$

$\mathrm{H} 46 \mathrm{~A}-\mathrm{C} 46-\mathrm{H} 46 \mathrm{~B}$

$\mathrm{C} 40-\mathrm{C} 46-\mathrm{H} 46 \mathrm{C}$

$\mathrm{H} 46 \mathrm{~A}-\mathrm{C} 46-\mathrm{H} 46 \mathrm{C}$

$\mathrm{H} 46 \mathrm{~B}-\mathrm{C} 46-\mathrm{H} 46 \mathrm{C}$

$\mathrm{C} 52-\mathrm{C} 47-\mathrm{C} 48$

$\mathrm{C} 52-\mathrm{C} 47-\mathrm{P} 2$

$\mathrm{C} 48-\mathrm{C} 47-\mathrm{P} 2$

$\mathrm{C} 49-\mathrm{C} 48-\mathrm{C} 47$

$\mathrm{C} 49-\mathrm{C} 48-\mathrm{C} 53$

$\mathrm{C} 47-\mathrm{C} 48-\mathrm{C} 53$

$\mathrm{C} 50-\mathrm{C} 49-\mathrm{C} 48$

$\mathrm{C} 50-\mathrm{C} 49-\mathrm{H} 49$

$\mathrm{C} 48-\mathrm{C} 49-\mathrm{H} 49$

$\mathrm{C} 49-\mathrm{C} 50-\mathrm{C} 51$

$\mathrm{C} 49-\mathrm{C} 50-\mathrm{H} 50$

C51- C50-H50

C52-C51-C50

C52-C51-H51

$\mathrm{C} 50-\mathrm{C} 51-\mathrm{H} 51$

$\mathrm{C} 51-\mathrm{C} 52-\mathrm{C} 47$

C $51-\mathrm{C} 52-\mathrm{H} 52$

$\mathrm{C} 47-\mathrm{C} 52-\mathrm{H} 52$

C54-C53-C58

$\mathrm{C} 54-\mathrm{C} 53-\mathrm{C} 48$

$\mathrm{C} 58-\mathrm{C} 53-\mathrm{C} 48$

$\mathrm{O} 3-\mathrm{C} 54-\mathrm{C} 53$

$\mathrm{O} 3-\mathrm{C} 54-\mathrm{C} 55$

C53-C54-C55

C56-C55-C54

C 56- C55-H55

C54-C55-H55

C57-C56-C55

C $57-\mathrm{C} 56-\mathrm{H} 56$

C55-C56-H56

C56-C57-C58

$\mathrm{C} 56-\mathrm{C} 57-\mathrm{H} 57$

C58-C57-H57
109.5

109.5

109.5

109.5

109.5

109.5

109.5

109.5

109.5

109.5

109.5

109.5

109.5

109.5

109.5

117.4 (4)

$111.3(3)$

$131.3(3)$

$118.2(4)$

113.5 (3)

128.3 (4)

123.2 (4)

118.4

118.4

118.8 (4)

120.6

120.6

119.4 (4)

120.3

120.3

123.0 (4)

118.5

118.5

118.3 (4)

122.5 (4)

118.5 (3)

115.7 (3)

123.4 (4)

120.9 (4)

118.2 (4)

120.9

120.9

122.5 (4)

118.7

118.7

118.3 (4)

120.9

120.9 


\begin{tabular}{|c|c|c|c|}
\hline $\mathrm{O} 2-\mathrm{C} 22-\mathrm{C} 21$ & $123.6(3)$ & $\mathrm{O} 4-\mathrm{C} 58-\mathrm{C} 57$ & $123.9(4)$ \\
\hline $\mathrm{O} 2-\mathrm{C} 22-\mathrm{C} 17$ & $115.0(3)$ & $\mathrm{O} 4-\mathrm{C} 58-\mathrm{C} 53$ & $114.3(4)$ \\
\hline $\mathrm{C} 21-\mathrm{C} 22-\mathrm{C} 17$ & $121.5(4)$ & $\mathrm{C} 57-\mathrm{C} 58-\mathrm{C} 53$ & $121.9(4)$ \\
\hline $\mathrm{O} 1-\mathrm{C} 23-\mathrm{H} 23 \mathrm{~A}$ & 109.5 & $\mathrm{O} 3-\mathrm{C} 59-\mathrm{H} 59 \mathrm{~A}$ & 109.5 \\
\hline $\mathrm{O} 1-\mathrm{C} 23-\mathrm{H} 23 \mathrm{~B}$ & 109.5 & $\mathrm{O} 3-\mathrm{C} 59-\mathrm{H} 59 \mathrm{~B}$ & 109.5 \\
\hline $\mathrm{H} 23 \mathrm{~A}-\mathrm{C} 23-\mathrm{H} 23 \mathrm{~B}$ & 109.5 & $\mathrm{H} 59 \mathrm{~A}-\mathrm{C} 59-\mathrm{H} 59 \mathrm{~B}$ & 109.5 \\
\hline $\mathrm{O} 1-\mathrm{C} 23-\mathrm{H} 23 \mathrm{C}$ & 109.5 & $\mathrm{O} 3-\mathrm{C} 59-\mathrm{H} 59 \mathrm{C}$ & 109.5 \\
\hline $\mathrm{H} 23 \mathrm{~A}-\mathrm{C} 23-\mathrm{H} 23 \mathrm{C}$ & 109.5 & $\mathrm{H} 59 \mathrm{~A}-\mathrm{C} 59-\mathrm{H} 59 \mathrm{C}$ & 109.5 \\
\hline $\mathrm{H} 23 \mathrm{~B}-\mathrm{C} 23-\mathrm{H} 23 \mathrm{C}$ & 109.5 & $\mathrm{H} 59 \mathrm{~B}-\mathrm{C} 59-\mathrm{H} 59 \mathrm{C}$ & 109.5 \\
\hline $\mathrm{O} 2-\mathrm{C} 24-\mathrm{H} 24 \mathrm{~A}$ & 109.5 & $\mathrm{O} 4-\mathrm{C} 60-\mathrm{H} 60 \mathrm{~A}$ & 109.5 \\
\hline $\mathrm{O} 2-\mathrm{C} 24-\mathrm{H} 24 \mathrm{~B}$ & 109.5 & $\mathrm{O} 4-\mathrm{C} 60-\mathrm{H} 60 \mathrm{~B}$ & 109.5 \\
\hline $\mathrm{H} 24 \mathrm{~A}-\mathrm{C} 24-\mathrm{H} 24 \mathrm{~B}$ & 109.5 & $\mathrm{H} 60 \mathrm{~A}-\mathrm{C} 60-\mathrm{H} 60 \mathrm{~B}$ & 109.5 \\
\hline $\mathrm{O} 2-\mathrm{C} 24-\mathrm{H} 24 \mathrm{C}$ & 109.5 & $\mathrm{O} 4-\mathrm{C} 60-\mathrm{H} 60 \mathrm{C}$ & 109.5 \\
\hline $\mathrm{H} 24 \mathrm{~A}-\mathrm{C} 24-\mathrm{H} 24 \mathrm{C}$ & 109.5 & $\mathrm{H} 60 \mathrm{~A}-\mathrm{C} 60-\mathrm{H} 60 \mathrm{C}$ & 109.5 \\
\hline $\mathrm{H} 24 \mathrm{~B}-\mathrm{C} 24-\mathrm{H} 24 \mathrm{C}$ & 109.5 & $\mathrm{H} 60 \mathrm{~B}-\mathrm{C} 60-\mathrm{H} 60 \mathrm{C}$ & 109.5 \\
\hline $\mathrm{C} 26-\mathrm{C} 25-\mathrm{C} 30$ & $111.3(3)$ & $\mathrm{C} 62-\mathrm{C} 61-\mathrm{C} 66$ & $111.1(3)$ \\
\hline $\mathrm{C} 26-\mathrm{C} 25-\mathrm{P} 1$ & $116.1(2)$ & $\mathrm{C} 62-\mathrm{C} 61-\mathrm{P} 2$ & $112.9(3)$ \\
\hline $\mathrm{C} 30-\mathrm{C} 25-\mathrm{P} 1$ & $116.8(3)$ & $\mathrm{C} 66-\mathrm{C} 61-\mathrm{P} 2$ & $118.4(3)$ \\
\hline $\mathrm{C} 26-\mathrm{C} 25-\mathrm{H} 25$ & 103.5 & $\mathrm{C} 62-\mathrm{C} 61-\mathrm{H} 61$ & 104.3 \\
\hline $\mathrm{C} 30-\mathrm{C} 25-\mathrm{H} 25$ & 103.5 & $\mathrm{C} 66-\mathrm{C} 61-\mathrm{H} 61$ & 104.3 \\
\hline $\mathrm{P} 1-\mathrm{C} 25-\mathrm{H} 25$ & 103.5 & $\mathrm{P} 2-\mathrm{C} 61-\mathrm{H} 61$ & 104.3 \\
\hline $\mathrm{C} 27-\mathrm{C} 26-\mathrm{C} 25$ & $108.2(3)$ & $\mathrm{C} 63-\mathrm{C} 62-\mathrm{C} 61$ & $109.6(3)$ \\
\hline $\mathrm{C} 27-\mathrm{C} 26-\mathrm{H} 26 \mathrm{~A}$ & 110.1 & $\mathrm{C} 63-\mathrm{C} 62-\mathrm{H} 62 \mathrm{~A}$ & 109.8 \\
\hline $\mathrm{C} 25-\mathrm{C} 26-\mathrm{H} 26 \mathrm{~A}$ & 110.1 & $\mathrm{C} 61-\mathrm{C} 62-\mathrm{H} 62 \mathrm{~A}$ & 109.8 \\
\hline $\mathrm{C} 27-\mathrm{C} 26-\mathrm{H} 26 \mathrm{~B}$ & 110.1 & С63-C62-H62B & 109.8 \\
\hline $\mathrm{C} 25-\mathrm{C} 26-\mathrm{H} 26 \mathrm{~B}$ & 110.1 & C61-C62-H62B & 109.8 \\
\hline $\mathrm{H} 26 \mathrm{~A}-\mathrm{C} 26-\mathrm{H} 26 \mathrm{~B}$ & 108.4 & $\mathrm{H} 62 \mathrm{~A}-\mathrm{C} 62-\mathrm{H} 62 \mathrm{~B}$ & 108.2 \\
\hline $\mathrm{C} 26-\mathrm{C} 27-\mathrm{C} 28$ & $111.9(3)$ & $\mathrm{C} 62-\mathrm{C} 63-\mathrm{C} 64$ & $110.9(4)$ \\
\hline $\mathrm{C} 26-\mathrm{C} 27-\mathrm{H} 27 \mathrm{~A}$ & 109.2 & $\mathrm{C} 62-\mathrm{C} 63-\mathrm{H} 63 \mathrm{~A}$ & 109.5 \\
\hline $\mathrm{C} 28-\mathrm{C} 27-\mathrm{H} 27 \mathrm{~A}$ & 109.2 & C64-C63-H63A & 109.5 \\
\hline $\mathrm{C} 26-\mathrm{C} 27-\mathrm{H} 27 \mathrm{~B}$ & 109.2 & C62-C63-H63B & 109.5 \\
\hline $\mathrm{C} 28-\mathrm{C} 27-\mathrm{H} 27 \mathrm{~B}$ & 109.2 & C64-C63-H63B & 109.5 \\
\hline $\mathrm{H} 27 \mathrm{~A}-\mathrm{C} 27-\mathrm{H} 27 \mathrm{~B}$ & 107.9 & $\mathrm{H} 63 \mathrm{~A}-\mathrm{C} 63-\mathrm{H} 63 \mathrm{~B}$ & 108.0 \\
\hline $\mathrm{C} 29-\mathrm{C} 28-\mathrm{C} 27$ & $113.0(3)$ & C65-C64-C63 & $111.2(4)$ \\
\hline $\mathrm{C} 29-\mathrm{C} 28-\mathrm{H} 28 \mathrm{~A}$ & 109.0 & C65-C64-H64A & 109.4 \\
\hline $\mathrm{C} 27-\mathrm{C} 28-\mathrm{H} 28 \mathrm{~A}$ & 109.0 & C63-C64-H64A & 109.4 \\
\hline $\mathrm{C} 29-\mathrm{C} 28-\mathrm{H} 28 \mathrm{~B}$ & 109.0 & C65-C64-H64B & 109.4 \\
\hline $\mathrm{C} 27-\mathrm{C} 28-\mathrm{H} 28 \mathrm{~B}$ & 109.0 & C63-C64-H64B & 109.4 \\
\hline $\mathrm{H} 28 \mathrm{~A}-\mathrm{C} 28-\mathrm{H} 28 \mathrm{~B}$ & 107.8 & $\mathrm{H} 64 \mathrm{~A}-\mathrm{C} 64-\mathrm{H} 64 \mathrm{~B}$ & 108.0 \\
\hline $\mathrm{C} 30-\mathrm{C} 29-\mathrm{C} 28$ & $111.1(3)$ & $\mathrm{C} 64-\mathrm{C} 65-\mathrm{C} 66$ & $111.7(4)$ \\
\hline $\mathrm{C} 30-\mathrm{C} 29-\mathrm{H} 29 \mathrm{~A}$ & 109.4 & $\mathrm{C} 64-\mathrm{C} 65-\mathrm{H} 65 \mathrm{~A}$ & 109.3 \\
\hline $\mathrm{C} 28-\mathrm{C} 29-\mathrm{H} 29 \mathrm{~A}$ & 109.4 & $\mathrm{C} 66-\mathrm{C} 65-\mathrm{H} 65 \mathrm{~A}$ & 109.3 \\
\hline $\mathrm{C} 30-\mathrm{C} 29-\mathrm{H} 29 \mathrm{~B}$ & 109.4 & C64-C65-H65B & 109.3 \\
\hline $\mathrm{C} 28-\mathrm{C} 29-\mathrm{H} 29 \mathrm{~B}$ & 109.4 & C66- $665-\mathrm{H} 65 \mathrm{~B}$ & 109.3 \\
\hline $\mathrm{H} 29 \mathrm{~A}-\mathrm{C} 29-\mathrm{H} 29 \mathrm{~B}$ & 108.0 & $\mathrm{H} 65 \mathrm{~A}-\mathrm{C} 65-\mathrm{H} 65 \mathrm{~B}$ & 107.9 \\
\hline $\mathrm{C} 29-\mathrm{C} 30-\mathrm{C} 25$ & $108.5(3)$ & $\mathrm{C} 65-\mathrm{C} 66-\mathrm{C} 61$ & $108.7(4)$ \\
\hline $\mathrm{C} 29-\mathrm{C} 30-\mathrm{H} 30 \mathrm{~A}$ & 110.0 & C65-C66-H66A & 109.9 \\
\hline $\mathrm{C} 25-\mathrm{C} 30-\mathrm{H} 30 \mathrm{~A}$ & 110.0 & C61-C66-H66A & 109.9 \\
\hline
\end{tabular}


$\mathrm{C} 29-\mathrm{C} 30-\mathrm{H} 30 \mathrm{~B}$

$\mathrm{C} 25-\mathrm{C} 30-\mathrm{H} 30 \mathrm{~B}$

$\mathrm{H} 30 \mathrm{~A}-\mathrm{C} 30-\mathrm{H} 30 \mathrm{~B}$

C32-C $31-\mathrm{C} 36$

$\mathrm{C} 32-\mathrm{C} 31-\mathrm{P} 1$

$\mathrm{C} 36-\mathrm{C} 31-\mathrm{P} 1$

$\mathrm{C} 32-\mathrm{C} 31-\mathrm{H} 31$

$\mathrm{C} 36-\mathrm{C} 31-\mathrm{H} 31$

$\mathrm{P} 1-\mathrm{C} 31-\mathrm{H} 31$

$\mathrm{C} 31-\mathrm{C} 32-\mathrm{C} 33$

$\mathrm{C} 31-\mathrm{C} 32-\mathrm{H} 32 \mathrm{~A}$

$\mathrm{C} 33-\mathrm{C} 32-\mathrm{H} 32 \mathrm{~A}$

$\mathrm{C} 31-\mathrm{C} 32-\mathrm{H} 32 \mathrm{~B}$

C $33-\mathrm{C} 32-\mathrm{H} 32 \mathrm{~B}$

$\mathrm{H} 32 \mathrm{~A}-\mathrm{C} 32-\mathrm{H} 32 \mathrm{~B}$

$\mathrm{C} 34-\mathrm{C} 33-\mathrm{C} 32$

C34-C $33-\mathrm{H} 33 \mathrm{~A}$

$\mathrm{C} 32-\mathrm{C} 33-\mathrm{H} 33 \mathrm{~A}$

C34-C33-H33B

C $32-\mathrm{C} 33-\mathrm{H} 33 \mathrm{~B}$

$\mathrm{H} 33 \mathrm{~A}-\mathrm{C} 33-\mathrm{H} 33 \mathrm{~B}$

$\mathrm{C} 35-\mathrm{C} 34-\mathrm{C} 33$

C35- C34-H34A

$\mathrm{C} 33-\mathrm{C} 34-\mathrm{H} 34 \mathrm{~A}$

C $35-\mathrm{C} 34-\mathrm{H} 34 \mathrm{~B}$

C33-C $34-\mathrm{H} 34 \mathrm{~B}$

H34A-C34-H34B

C34-C $35-\mathrm{C} 36$

C34-C $35-\mathrm{H} 35 \mathrm{~A}$

$\mathrm{C} 36-\mathrm{C} 35-\mathrm{H} 35 \mathrm{~A}$

$\mathrm{C} 34-\mathrm{C} 35-\mathrm{H} 35 \mathrm{~B}$

C $36-\mathrm{C} 35-\mathrm{H} 35 \mathrm{~B}$

$\mathrm{H} 35 \mathrm{~A}-\mathrm{C} 35-\mathrm{H} 35 \mathrm{~B}$

$\mathrm{C} 35-\mathrm{C} 36-\mathrm{C} 31$

$\mathrm{C} 35-\mathrm{C} 36-\mathrm{H} 36 \mathrm{~A}$

C31-C $36-\mathrm{H} 36 \mathrm{~A}$

C35-C $36-\mathrm{H} 36 \mathrm{~B}$

$\mathrm{C} 31-\mathrm{C} 36-\mathrm{H} 36 \mathrm{~B}$

$\mathrm{H} 36 \mathrm{~A}-\mathrm{C} 36-\mathrm{H} 36 \mathrm{~B}$

$\mathrm{C} 6-\mathrm{C} 1-\mathrm{C} 2-\mathrm{C} 3$

$\mathrm{C} 7-\mathrm{C} 1-\mathrm{C} 2-\mathrm{C} 3$

$\mathrm{Ru} 1-\mathrm{C} 1-\mathrm{C} 2-\mathrm{C} 3$

$\mathrm{C} 6-\mathrm{C} 1-\mathrm{C} 2-\mathrm{Ru} 1$

$\mathrm{C} 7-\mathrm{C} 1-\mathrm{C} 2-\mathrm{Ru} 1$

$\mathrm{C} 1-\mathrm{C} 2-\mathrm{C} 3-\mathrm{C} 4$

$\mathrm{Ru} 1-\mathrm{C} 2-\mathrm{C} 3-\mathrm{C} 4$

$\mathrm{C} 1-\mathrm{C} 2-\mathrm{C} 3-\mathrm{Ru} 1$
110.0

110.0

108.4

109.3 (3)

$111.4(2)$

113.8 (2)

107.3

107.3

107.3

112.3 (3)

109.1

109.1

109.1

109.1

107.9

$110.6(3)$

109.5

109.5

109.5

109.5

108.1

110.1 (3)

109.6

109.6

109.6

109.6

108.1

$112.6(3)$

109.1

109.1

109.1

109.1

107.8

$110.1(3)$

109.6

109.6

109.6

109.6

108.1

$1.6(5)$

$-170.8(3)$

54.8 (3)

$-53.2(3)$

$134.4(3)$

$-2.0(5)$

$52.5(3)$

$-54.5(3)$
C65-C66-H66B

C61-C66-H66B

H66A-C66-H66B

C68-C67-C72

C68-C67-P2

$\mathrm{C} 72-\mathrm{C} 67-\mathrm{P} 2$

C68-C67-H67

C72-C67-H67

P2-C67-H67

C67-C68-C69

C67-C68-H68A

C69- C68-H68A

C67-C68-H68B

C69- $668-\mathrm{H} 68 \mathrm{~B}$

$\mathrm{H} 68 \mathrm{~A}-\mathrm{C} 68-\mathrm{H} 68 \mathrm{~B}$

$\mathrm{C} 70-\mathrm{C} 69-\mathrm{C} 68$

$\mathrm{C} 70-\mathrm{C} 69-\mathrm{H} 69 \mathrm{~A}$

C68-C69-H69A

C70- 6 69-H69B

C68-C69- $699 \mathrm{~B}$

H69A-C69-H69B

C71-C70-C69

C71-C70-H70A

$\mathrm{C} 69-\mathrm{C} 70-\mathrm{H} 70 \mathrm{~A}$

C71- C70-H70B

C69- $\mathrm{C} 70-\mathrm{H} 70 \mathrm{~B}$

$\mathrm{H} 70 \mathrm{~A}-\mathrm{C} 70-\mathrm{H} 70 \mathrm{~B}$

$\mathrm{C} 70-\mathrm{C} 71-\mathrm{C} 72$

C70-C71-H71A

C72-C71-H71A

C70-C71-H71B

C72-C71-H71B

$\mathrm{H} 71 \mathrm{~A}-\mathrm{C} 71-\mathrm{H} 71 \mathrm{~B}$

C71-C72-C67

$\mathrm{C} 71-\mathrm{C} 72-\mathrm{H} 72 \mathrm{~A}$

$\mathrm{C} 67-\mathrm{C} 72-\mathrm{H} 72 \mathrm{~A}$

$\mathrm{C} 71-\mathrm{C} 72-\mathrm{H} 72 \mathrm{~B}$

$\mathrm{C} 67-\mathrm{C} 72-\mathrm{H} 72 \mathrm{~B}$

$\mathrm{H} 72 \mathrm{~A}-\mathrm{C} 72-\mathrm{H} 72 \mathrm{~B}$

$\mathrm{C} 42-\mathrm{C} 37-\mathrm{C} 38-\mathrm{C} 39$

$\mathrm{C} 43-\mathrm{C} 37-\mathrm{C} 38-\mathrm{C} 39$

$\mathrm{Ru} 2-\mathrm{C} 37-\mathrm{C} 38-\mathrm{C} 39$

$\mathrm{C} 42-\mathrm{C} 37-\mathrm{C} 38-\mathrm{Ru} 2$

$\mathrm{C} 43-\mathrm{C} 37-\mathrm{C} 38-\mathrm{Ru} 2$

C $37-\mathrm{C} 38-\mathrm{C} 39-\mathrm{C} 40$

$\mathrm{Ru} 2-\mathrm{C} 38-\mathrm{C} 39-\mathrm{C} 40$

C37-C38-C39-Ru2
109.9

109.9

108.3

$109.8(4)$

$110.9(3)$

$114.0(3)$

107.3

107.3

107.3

111.7 (4)

109.3

109.3

109.3

109.3

108.0

110.0 (4)

109.7

109.7

109.7

109.7

108.2

$110.9(4)$

109.5

109.5

109.5

109.5

108.0

113.4 (4)

108.9

108.9

108.9

108.9

107.7

110.5 (4)

109.5

109.5

109.5

109.5

108.1

$0.0(5)$

$170.0(3)$

-54.2 (3)

54.2 (3)

-135.8 (3)

$1.2(6)$

$-52.9(3)$

$54.1(3)$ 


\begin{tabular}{|c|c|}
\hline $\mathrm{C} 2-\mathrm{C} 3-\mathrm{C} 4-\mathrm{C} 5$ & $2.2(5)$ \\
\hline $\mathrm{Ru} 1-\mathrm{C} 3-\mathrm{C} 4-\mathrm{C} 5$ & $55.0(3)$ \\
\hline $\mathrm{C} 2-\mathrm{C} 3-\mathrm{C} 4-\mathrm{C} 10$ & $-179.3(3)$ \\
\hline $\mathrm{Ru} 1-\mathrm{C} 3-\mathrm{C} 4-\mathrm{C} 10$ & $-126.4(3)$ \\
\hline $\mathrm{C} 2-\mathrm{C} 3-\mathrm{C} 4-\mathrm{Ru} 1$ & $-52.8(3)$ \\
\hline $\mathrm{C} 3-\mathrm{C} 4-\mathrm{C} 5-\mathrm{C} 6$ & $-2.1(5)$ \\
\hline $\mathrm{C} 10-\mathrm{C} 4-\mathrm{C} 5-\mathrm{C} 6$ & $179.4(3)$ \\
\hline $\mathrm{Ru} 1-\mathrm{C} 4-\mathrm{C} 5-\mathrm{C} 6$ & $53.8(3)$ \\
\hline $\mathrm{C} 3-\mathrm{C} 4-\mathrm{C} 5-\mathrm{Ru} 1$ & $-55.9(3)$ \\
\hline $\mathrm{C} 10-\mathrm{C} 4-\mathrm{C} 5-\mathrm{Ru} 1$ & $125.5(3)$ \\
\hline $\mathrm{C} 2-\mathrm{C} 1-\mathrm{C} 6-\mathrm{C} 5$ & $-1.5(5)$ \\
\hline $\mathrm{C} 7-\mathrm{C} 1-\mathrm{C} 6-\mathrm{C} 5$ & $171.0(3)$ \\
\hline $\mathrm{Ru} 1-\mathrm{C} 1-\mathrm{C} 6-\mathrm{C} 5$ & $-55.6(3)$ \\
\hline $\mathrm{C} 2-\mathrm{C} 1-\mathrm{C} 6-\mathrm{Ru} 1$ & $54.1(3)$ \\
\hline $\mathrm{C} 7-\mathrm{C} 1-\mathrm{C} 6-\mathrm{Ru} 1$ & $-133.3(3)$ \\
\hline $\mathrm{C} 4-\mathrm{C} 5-\mathrm{C} 6-\mathrm{C} 1$ & $1.8(5)$ \\
\hline $\mathrm{Ru} 1-\mathrm{C} 5-\mathrm{C} 6-\mathrm{C} 1$ & $56.5(3)$ \\
\hline $\mathrm{C} 4-\mathrm{C} 5-\mathrm{C} 6-\mathrm{Ru} 1$ & $-54.7(3)$ \\
\hline $\mathrm{C} 6-\mathrm{C} 1-\mathrm{C} 7-\mathrm{C} 9$ & $149.8(4)$ \\
\hline $\mathrm{C} 2-\mathrm{C} 1-\mathrm{C} 7-\mathrm{C} 9$ & $-38.0(6)$ \\
\hline $\mathrm{Ru} 1-\mathrm{C} 1-\mathrm{C} 7-\mathrm{C} 9$ & $58.7(6)$ \\
\hline $\mathrm{C} 6-\mathrm{C} 1-\mathrm{C} 7-\mathrm{C} 8$ & $-86.2(4)$ \\
\hline $\mathrm{C} 2-\mathrm{C} 1-\mathrm{C} 7-\mathrm{C} 8$ & $86.0(4)$ \\
\hline $\mathrm{Ru} 1-\mathrm{C} 1-\mathrm{C} 7-\mathrm{C} 8$ & $-177.3(3)$ \\
\hline $\mathrm{C} 31-\mathrm{P} 1-\mathrm{C} 11-\mathrm{C} 12$ & $8.6(4)$ \\
\hline $\mathrm{C} 25-\mathrm{P} 1-\mathrm{C} 11-\mathrm{C} 12$ & $118.7(4)$ \\
\hline $\mathrm{Ru} 1-\mathrm{P} 1-\mathrm{C} 11-\mathrm{C} 12$ & $-122.0(3)$ \\
\hline $\mathrm{C} 31-\mathrm{P} 1-\mathrm{C} 11-\mathrm{C} 16$ & $-169.5(3)$ \\
\hline $\mathrm{C} 25-\mathrm{P} 1-\mathrm{C} 11-\mathrm{C} 16$ & $-59.4(3)$ \\
\hline $\mathrm{Ru} 1-\mathrm{P} 1-\mathrm{C} 11-\mathrm{C} 16$ & $59.8(3)$ \\
\hline $\mathrm{C} 16-\mathrm{C} 11-\mathrm{C} 12-\mathrm{C} 13$ & $4.5(5)$ \\
\hline $\mathrm{P} 1-\mathrm{C} 11-\mathrm{C} 12-\mathrm{C} 13$ & $-173.6(3)$ \\
\hline $\mathrm{C} 16-\mathrm{C} 11-\mathrm{C} 12-\mathrm{C} 17$ & $-173.0(3)$ \\
\hline $\mathrm{P} 1-\mathrm{C} 11-\mathrm{C} 12-\mathrm{C} 17$ & $9.0(6)$ \\
\hline $\mathrm{C} 11-\mathrm{C} 12-\mathrm{C} 13-\mathrm{C} 14$ & $-2.1(6)$ \\
\hline $\mathrm{C} 17-\mathrm{C} 12-\mathrm{C} 13-\mathrm{C} 14$ & $175.7(4)$ \\
\hline $\mathrm{C} 12-\mathrm{C} 13-\mathrm{C} 14-\mathrm{C} 15$ & $-1.1(6)$ \\
\hline $\mathrm{C} 13-\mathrm{C} 14-\mathrm{C} 15-\mathrm{C} 16$ & $1.8(6)$ \\
\hline $\mathrm{C} 14-\mathrm{C} 15-\mathrm{C} 16-\mathrm{C} 11$ & $0.7(6)$ \\
\hline $\mathrm{C} 12-\mathrm{C} 11-\mathrm{C} 16-\mathrm{C} 15$ & $-3.9(5)$ \\
\hline $\mathrm{P} 1-\mathrm{C} 11-\mathrm{C} 16-\mathrm{C} 15$ & $174.5(3)$ \\
\hline $\mathrm{C} 11-\mathrm{C} 12-\mathrm{C} 17-\mathrm{C} 18$ & $-95.8(5)$ \\
\hline $\mathrm{C} 13-\mathrm{C} 12-\mathrm{C} 17-\mathrm{C} 18$ & $86.7(5)$ \\
\hline $\mathrm{C} 11-\mathrm{C} 12-\mathrm{C} 17-\mathrm{C} 22$ & $92.6(5)$ \\
\hline $\mathrm{C} 13-\mathrm{C} 12-\mathrm{C} 17-\mathrm{C} 22$ & $-85.0(5)$ \\
\hline $\mathrm{C} 23-\mathrm{O} 1-\mathrm{C} 18-\mathrm{C} 17$ & $167.6(4)$ \\
\hline $\mathrm{C} 23-\mathrm{O} 1-\mathrm{C} 18-\mathrm{C} 19$ & $-11.5(5)$ \\
\hline $\mathrm{C} 22-\mathrm{C} 17-\mathrm{C} 18-\mathrm{O} 1$ & $-179.3(3)$ \\
\hline
\end{tabular}

\begin{tabular}{|c|c|}
\hline $\mathrm{C} 38-\mathrm{C} 39-\mathrm{C} 40-\mathrm{C} 41$ & $-2.0(6)$ \\
\hline $\mathrm{Ru} 2-\mathrm{C} 39-\mathrm{C} 40-\mathrm{C} 41$ & $-55.6(3)$ \\
\hline $\mathrm{C} 38-\mathrm{C} 39-\mathrm{C} 40-\mathrm{C} 46$ & $178.1(4)$ \\
\hline $\mathrm{Ru} 2-\mathrm{C} 39-\mathrm{C} 40-\mathrm{C} 46$ & $124.5(4)$ \\
\hline $\mathrm{C} 38-\mathrm{C} 39-\mathrm{C} 40-\mathrm{Ru} 2$ & $53.6(3)$ \\
\hline $\mathrm{C} 39-\mathrm{C} 40-\mathrm{C} 41-\mathrm{C} 42$ & $1.7(6)$ \\
\hline $\mathrm{C} 46-\mathrm{C} 40-\mathrm{C} 41-\mathrm{C} 42$ & $-178.4(4)$ \\
\hline $\mathrm{Ru} 2-\mathrm{C} 40-\mathrm{C} 41-\mathrm{C} 42$ & $-54.5(3)$ \\
\hline $\mathrm{C} 39-\mathrm{C} 40-\mathrm{C} 41-\mathrm{Ru} 2$ & $56.2(3)$ \\
\hline $\mathrm{C} 46-\mathrm{C} 40-\mathrm{C} 41-\mathrm{Ru} 2$ & $-123.9(4)$ \\
\hline $\mathrm{C} 38-\mathrm{C} 37-\mathrm{C} 42-\mathrm{C} 41$ & $-0.3(5)$ \\
\hline $\mathrm{C} 43-\mathrm{C} 37-\mathrm{C} 42-\mathrm{C} 41$ & $-170.2(4)$ \\
\hline $\mathrm{Ru} 2-\mathrm{C} 37-\mathrm{C} 42-\mathrm{C} 41$ & $54.4(3)$ \\
\hline $\mathrm{C} 38-\mathrm{C} 37-\mathrm{C} 42-\mathrm{Ru} 2$ & $-54.7(3)$ \\
\hline $\mathrm{C} 43-\mathrm{C} 37-\mathrm{C} 42-\mathrm{Ru} 2$ & $135.4(4)$ \\
\hline $\mathrm{C} 40-\mathrm{C} 41-\mathrm{C} 42-\mathrm{C} 37$ & $-0.6(6)$ \\
\hline $\mathrm{Ru} 2-\mathrm{C} 41-\mathrm{C} 42-\mathrm{C} 37$ & $-55.6(3)$ \\
\hline $\mathrm{C} 40-\mathrm{C} 41-\mathrm{C} 42-\mathrm{Ru} 2$ & $55.0(3)$ \\
\hline $\mathrm{C} 42-\mathrm{C} 37-\mathrm{C} 43-\mathrm{C} 45$ & $-148.6(4)$ \\
\hline $\mathrm{C} 38-\mathrm{C} 37-\mathrm{C} 43-\mathrm{C} 45$ & $41.8(5)$ \\
\hline $\mathrm{Ru} 2-\mathrm{C} 37-\mathrm{C} 43-\mathrm{C} 45$ & $-53.4(6)$ \\
\hline $\mathrm{C} 42-\mathrm{C} 37-\mathrm{C} 43-\mathrm{C} 44$ & $88.6(5)$ \\
\hline $\mathrm{C} 38-\mathrm{C} 37-\mathrm{C} 43-\mathrm{C} 44$ & $-81.0(5)$ \\
\hline $\mathrm{Ru} 2-\mathrm{C} 37-\mathrm{C} 43-\mathrm{C} 44$ & $-176.3(4)$ \\
\hline C67-P2-C47-C52 & $173.9(3)$ \\
\hline $\mathrm{C} 61-\mathrm{P} 2-\mathrm{C} 47-\mathrm{C} 52$ & $64.2(3)$ \\
\hline $\mathrm{Ru} 2-\mathrm{P} 2-\mathrm{C} 47-\mathrm{C} 52$ & $-57.7(3)$ \\
\hline $\mathrm{C} 67-\mathrm{P} 2-\mathrm{C} 47-\mathrm{C} 48$ & $-6.9(4)$ \\
\hline $\mathrm{C} 61-\mathrm{P} 2-\mathrm{C} 47-\mathrm{C} 48$ & $-116.7(4)$ \\
\hline $\mathrm{Ru} 2-\mathrm{P} 2-\mathrm{C} 47-\mathrm{C} 48$ & $121.4(4)$ \\
\hline $\mathrm{C} 52-\mathrm{C} 47-\mathrm{C} 48-\mathrm{C} 49$ & $0.5(6)$ \\
\hline $\mathrm{P} 2-\mathrm{C} 47-\mathrm{C} 48-\mathrm{C} 49$ & $-178.5(3)$ \\
\hline $\mathrm{C} 52-\mathrm{C} 47-\mathrm{C} 48-\mathrm{C} 53$ & $177.4(4)$ \\
\hline $\mathrm{P} 2-\mathrm{C} 47-\mathrm{C} 48-\mathrm{C} 53$ & $-1.7(6)$ \\
\hline $\mathrm{C} 47-\mathrm{C} 48-\mathrm{C} 49-\mathrm{C} 50$ & $-0.4(6)$ \\
\hline $\mathrm{C} 53-\mathrm{C} 48-\mathrm{C} 49-\mathrm{C} 50$ & $-177.8(4)$ \\
\hline $\mathrm{C} 48-\mathrm{C} 49-\mathrm{C} 50-\mathrm{C} 51$ & $0.0(6)$ \\
\hline $\mathrm{C} 49-\mathrm{C} 50-\mathrm{C} 51-\mathrm{C} 52$ & $0.3(6)$ \\
\hline $\mathrm{C} 50-\mathrm{C} 51-\mathrm{C} 52-\mathrm{C} 47$ & $-0.2(7)$ \\
\hline $\mathrm{C} 48-\mathrm{C} 47-\mathrm{C} 52-\mathrm{C} 51$ & $-0.2(6)$ \\
\hline $\mathrm{P} 2-\mathrm{C} 47-\mathrm{C} 52-\mathrm{C} 51$ & $179.0(3)$ \\
\hline $\mathrm{C} 49-\mathrm{C} 48-\mathrm{C} 53-\mathrm{C} 54$ & $-94.7(5)$ \\
\hline $\mathrm{C} 47-\mathrm{C} 48-\mathrm{C} 53-\mathrm{C} 54$ & $88.3(5)$ \\
\hline $\mathrm{C} 49-\mathrm{C} 48-\mathrm{C} 53-\mathrm{C} 58$ & $75.2(5)$ \\
\hline $\mathrm{C} 47-\mathrm{C} 48-\mathrm{C} 53-\mathrm{C} 58$ & $-101.8(5)$ \\
\hline $\mathrm{C} 59-\mathrm{O} 3-\mathrm{C} 54-\mathrm{C} 53$ & $-163.0(3)$ \\
\hline $\mathrm{C} 59-\mathrm{O} 3-\mathrm{C} 54-\mathrm{C} 55$ & $16.5(6)$ \\
\hline $\mathrm{C} 58-\mathrm{C} 53-\mathrm{C} 54-\mathrm{O} 3$ & $-179.2(4)$ \\
\hline
\end{tabular}




\begin{tabular}{|c|c|}
\hline $\mathrm{C} 12-\mathrm{C} 17-\mathrm{C} 18-\mathrm{O} 1$ & $8.9(5)$ \\
\hline $\mathrm{C} 22-\mathrm{C} 17-\mathrm{C} 18-\mathrm{C} 19$ & $-0.2(6)$ \\
\hline $\mathrm{C} 12-\mathrm{C} 17-\mathrm{C} 18-\mathrm{C} 19$ & $-172.0(4)$ \\
\hline $\mathrm{O} 1-\mathrm{C} 18-\mathrm{C} 19-\mathrm{C} 20$ & $179.3(4)$ \\
\hline $\mathrm{C} 17-\mathrm{C} 18-\mathrm{C} 19-\mathrm{C} 20$ & $0.2(6)$ \\
\hline $\mathrm{C} 18-\mathrm{C} 19-\mathrm{C} 20-\mathrm{C} 21$ & $0.2(6)$ \\
\hline $\mathrm{C} 19-\mathrm{C} 20-\mathrm{C} 21-\mathrm{C} 22$ & $-0.6(7)$ \\
\hline $\mathrm{C} 24-\mathrm{O} 2-\mathrm{C} 22-\mathrm{C} 21$ & $2.0(6)$ \\
\hline $\mathrm{C} 24-\mathrm{O} 2-\mathrm{C} 22-\mathrm{C} 17$ & $-178.8(4)$ \\
\hline $\mathrm{C} 20-\mathrm{C} 21-\mathrm{C} 22-\mathrm{O} 2$ & $179.8(4)$ \\
\hline $\mathrm{C} 20-\mathrm{C} 21-\mathrm{C} 22-\mathrm{C} 17$ & $0.7(6)$ \\
\hline $\mathrm{C} 18-\mathrm{C} 17-\mathrm{C} 22-\mathrm{O} 2$ & $-179.5(3)$ \\
\hline $\mathrm{C} 12-\mathrm{C} 17-\mathrm{C} 22-\mathrm{O} 2$ & $-7.5(5)$ \\
\hline $\mathrm{C} 18-\mathrm{C} 17-\mathrm{C} 22-\mathrm{C} 21$ & $-0.3(6)$ \\
\hline $\mathrm{C} 12-\mathrm{C} 17-\mathrm{C} 22-\mathrm{C} 21$ & $171.7(4)$ \\
\hline $\mathrm{C} 11-\mathrm{P} 1-\mathrm{C} 25-\mathrm{C} 26$ & $-53.1(3)$ \\
\hline $\mathrm{C} 31-\mathrm{P} 1-\mathrm{C} 25-\mathrm{C} 26$ & $58.7(3)$ \\
\hline $\mathrm{Ru} 1-\mathrm{P} 1-\mathrm{C} 25-\mathrm{C} 26$ & $-166.9(2)$ \\
\hline $\mathrm{C} 11-\mathrm{P} 1-\mathrm{C} 25-\mathrm{C} 30$ & $172.4(3)$ \\
\hline $\mathrm{C} 31-\mathrm{P} 1-\mathrm{C} 25-\mathrm{C} 30$ & $-75.9(3)$ \\
\hline $\mathrm{Ru} 1-\mathrm{P} 1-\mathrm{C} 25-\mathrm{C} 30$ & $58.6(3)$ \\
\hline $\mathrm{C} 30-\mathrm{C} 25-\mathrm{C} 26-\mathrm{C} 27$ & $-61.2(4)$ \\
\hline $\mathrm{P} 1-\mathrm{C} 25-\mathrm{C} 26-\mathrm{C} 27$ & $161.8(3)$ \\
\hline $\mathrm{C} 25-\mathrm{C} 26-\mathrm{C} 27-\mathrm{C} 28$ & $55.3(4)$ \\
\hline $\mathrm{C} 26-\mathrm{C} 27-\mathrm{C} 28-\mathrm{C} 29$ & $-52.5(4)$ \\
\hline $\mathrm{C} 27-\mathrm{C} 28-\mathrm{C} 29-\mathrm{C} 30$ & $52.8(4)$ \\
\hline $\mathrm{C} 28-\mathrm{C} 29-\mathrm{C} 30-\mathrm{C} 25$ & $-56.3(4)$ \\
\hline $\mathrm{C} 26-\mathrm{C} 25-\mathrm{C} 30-\mathrm{C} 29$ & $62.2(4)$ \\
\hline $\mathrm{P} 1-\mathrm{C} 25-\mathrm{C} 30-\mathrm{C} 29$ & $-161.3(2)$ \\
\hline $\mathrm{C} 11-\mathrm{P} 1-\mathrm{C} 31-\mathrm{C} 32$ & $-92.0(3)$ \\
\hline $\mathrm{C} 25-\mathrm{P} 1-\mathrm{C} 31-\mathrm{C} 32$ & $164.6(3)$ \\
\hline $\mathrm{Ru} 1-\mathrm{P} 1-\mathrm{C} 31-\mathrm{C} 32$ & $32.3(3)$ \\
\hline $\mathrm{C} 11-\mathrm{P} 1-\mathrm{C} 31-\mathrm{C} 36$ & $143.8(3)$ \\
\hline $\mathrm{C} 25-\mathrm{P} 1-\mathrm{C} 31-\mathrm{C} 36$ & $40.4(3)$ \\
\hline $\mathrm{Ru} 1-\mathrm{P} 1-\mathrm{C} 31-\mathrm{C} 36$ & $-91.8(3)$ \\
\hline $\mathrm{C} 36-\mathrm{C} 31-\mathrm{C} 32-\mathrm{C} 33$ & $-57.2(4)$ \\
\hline $\mathrm{P} 1-\mathrm{C} 31-\mathrm{C} 32-\mathrm{C} 33$ & $176.2(3)$ \\
\hline $\mathrm{C} 31-\mathrm{C} 32-\mathrm{C} 33-\mathrm{C} 34$ & $57.2(5)$ \\
\hline $\mathrm{C} 32-\mathrm{C} 33-\mathrm{C} 34-\mathrm{C} 35$ & $-55.0(5)$ \\
\hline $\mathrm{C} 33-\mathrm{C} 34-\mathrm{C} 35-\mathrm{C} 36$ & $56.3(5)$ \\
\hline $\mathrm{C} 34-\mathrm{C} 35-\mathrm{C} 36-\mathrm{C} 31$ & $-57.0(5)$ \\
\hline $\mathrm{C} 32-\mathrm{C} 31-\mathrm{C} 36-\mathrm{C} 35$ & $56.0(4)$ \\
\hline $\mathrm{P} 1-\mathrm{C} 31-\mathrm{C} 36-\mathrm{C} 35$ & $-178.7(3)$ \\
\hline
\end{tabular}

\begin{tabular}{|c|c|}
\hline $\mathrm{C} 48-\mathrm{C} 53-\mathrm{C} 54-\mathrm{O} 3$ & $-9.2(6)$ \\
\hline $\mathrm{C} 58-\mathrm{C} 53-\mathrm{C} 54-\mathrm{C} 55$ & $1.3(6)$ \\
\hline $\mathrm{C} 48-\mathrm{C} 53-\mathrm{C} 54-\mathrm{C} 55$ & $171.2(4)$ \\
\hline $\mathrm{O} 3-\mathrm{C} 54-\mathrm{C} 55-\mathrm{C} 56$ & $179.2(4)$ \\
\hline $\mathrm{C} 53-\mathrm{C} 54-\mathrm{C} 55-\mathrm{C} 56$ & $-1.4(6)$ \\
\hline $\mathrm{C} 54-\mathrm{C} 55-\mathrm{C} 56-\mathrm{C} 57$ & $1.2(7)$ \\
\hline $\mathrm{C} 55-\mathrm{C} 56-\mathrm{C} 57-\mathrm{C} 58$ & $-1.0(7)$ \\
\hline $\mathrm{C} 60-\mathrm{O} 4-\mathrm{C} 58-\mathrm{C} 57$ & $5.3(6)$ \\
\hline $\mathrm{C} 60-\mathrm{O} 4-\mathrm{C} 58-\mathrm{C} 53$ & $-175.3(4)$ \\
\hline $\mathrm{C} 56-\mathrm{C} 57-\mathrm{C} 58-\mathrm{O} 4$ & $-179.7(4)$ \\
\hline $\mathrm{C} 56-\mathrm{C} 57-\mathrm{C} 58-\mathrm{C} 53$ & $1.0(7)$ \\
\hline $\mathrm{C} 54-\mathrm{C} 53-\mathrm{C} 58-\mathrm{O} 4$ & $179.5(4)$ \\
\hline $\mathrm{C} 48-\mathrm{C} 53-\mathrm{C} 58-\mathrm{O} 4$ & $9.1(5)$ \\
\hline $\mathrm{C} 54-\mathrm{C} 53-\mathrm{C} 58-\mathrm{C} 57$ & $-1.1(6)$ \\
\hline $\mathrm{C} 48-\mathrm{C} 53-\mathrm{C} 58-\mathrm{C} 57$ & $-171.4(4)$ \\
\hline C47-P2-C61-C62 & $57.1(3)$ \\
\hline C67-P2-C61-C62 & $-54.7(3)$ \\
\hline $\mathrm{Ru} 2-\mathrm{P} 2-\mathrm{C} 61-\mathrm{C} 62$ & $171.8(2)$ \\
\hline $\mathrm{C} 47-\mathrm{P} 2-\mathrm{C} 61-\mathrm{C} 66$ & $-170.7(3)$ \\
\hline C67-P2-C61-C66 & $77.6(3)$ \\
\hline $\mathrm{Ru} 2-\mathrm{P} 2-\mathrm{C} 61-\mathrm{C} 66$ & $-55.9(3)$ \\
\hline $\mathrm{C} 66-\mathrm{C} 61-\mathrm{C} 62-\mathrm{C} 63$ & $59.2(5)$ \\
\hline $\mathrm{P} 2-\mathrm{C} 61-\mathrm{C} 62-\mathrm{C} 63$ & $-165.2(3)$ \\
\hline $\mathrm{C} 61-\mathrm{C} 62-\mathrm{C} 63-\mathrm{C} 64$ & $-57.5(5)$ \\
\hline $\mathrm{C} 62-\mathrm{C} 63-\mathrm{C} 64-\mathrm{C} 65$ & $56.4(5)$ \\
\hline $\mathrm{C} 63-\mathrm{C} 64-\mathrm{C} 65-\mathrm{C} 66$ & $-56.2(5)$ \\
\hline $\mathrm{C} 64-\mathrm{C} 65-\mathrm{C} 66-\mathrm{C} 61$ & $56.3(4)$ \\
\hline $\mathrm{C} 62-\mathrm{C} 61-\mathrm{C} 66-\mathrm{C} 65$ & $-58.0(4)$ \\
\hline P2-C61-C66-C65 & $169.0(3)$ \\
\hline $\mathrm{C} 47-\mathrm{P} 2-\mathrm{C} 67-\mathrm{C} 68$ & $85.1(3)$ \\
\hline $\mathrm{C} 61-\mathrm{P} 2-\mathrm{C} 67-\mathrm{C} 68$ & $-171.3(3)$ \\
\hline $\mathrm{Ru} 2-\mathrm{P} 2-\mathrm{C} 67-\mathrm{C} 68$ & $-37.9(3)$ \\
\hline $\mathrm{C} 47-\mathrm{P} 2-\mathrm{C} 67-\mathrm{C} 72$ & $-150.4(3)$ \\
\hline $\mathrm{C} 61-\mathrm{P} 2-\mathrm{C} 67-\mathrm{C} 72$ & $-46.8(4)$ \\
\hline $\mathrm{Ru} 2-\mathrm{P} 2-\mathrm{C} 67-\mathrm{C} 72$ & $86.7(3)$ \\
\hline $\mathrm{C} 72-\mathrm{C} 67-\mathrm{C} 68-\mathrm{C} 69$ & $57.4(5)$ \\
\hline $\mathrm{P} 2-\mathrm{C} 67-\mathrm{C} 68-\mathrm{C} 69$ & $-175.7(3)$ \\
\hline $\mathrm{C} 67-\mathrm{C} 68-\mathrm{C} 69-\mathrm{C} 70$ & $-57.7(5)$ \\
\hline $\mathrm{C} 68-\mathrm{C} 69-\mathrm{C} 70-\mathrm{C} 71$ & $55.3(5)$ \\
\hline $\mathrm{C} 69-\mathrm{C} 70-\mathrm{C} 71-\mathrm{C} 72$ & $-55.2(5)$ \\
\hline $\mathrm{C} 70-\mathrm{C} 71-\mathrm{C} 72-\mathrm{C} 67$ & $54.9(5)$ \\
\hline $\mathrm{C} 68-\mathrm{C} 67-\mathrm{C} 72-\mathrm{C} 71$ & $-54.8(5)$ \\
\hline $\mathrm{P} 2-\mathrm{C} 67-\mathrm{C} 72-\mathrm{C} 71$ & $-179.9(3)$ \\
\hline
\end{tabular}


Hydrogen-bond geometry $\left(A,{ }^{\circ}\right)$

$\mathrm{Cg} 3$ and $\mathrm{Cg} 8$ are the centroids of rings $\mathrm{C} 17-\mathrm{C} 22$ and $\mathrm{C} 53-\mathrm{C} 58$, respectively.

\begin{tabular}{lllll}
\hline$D-\mathrm{H} \cdots A$ & $D-\mathrm{H}$ & $\mathrm{H} \cdots A$ & $D \cdots A$ & $D-\mathrm{H} \cdots A$ \\
\hline $\mathrm{C} 6-\mathrm{H} 6 \cdots \mathrm{O} 2$ & 0.95 & 2.50 & $3.328(6)$ & 146 \\
$\mathrm{C} 30-\mathrm{H} 30 A \cdots \mathrm{Cl} 2$ & 0.99 & 2.74 & $3.552(4)$ & 139 \\
$\mathrm{C} 45-\mathrm{H} 45 C \cdots \mathrm{Cl} 3$ & 0.98 & 2.79 & $3.577(5)$ & 137 \\
$\mathrm{C} 46-\mathrm{H} 46 C \cdots \mathrm{C} 14$ & 0.98 & 2.68 & $3.302(4)$ & 122 \\
$\mathrm{C} 62-\mathrm{H} 62 A \cdots \mathrm{O} 3$ & 0.99 & 2.58 & $3.261(6)$ & 126 \\
$\mathrm{C} 66-\mathrm{H} 66 B \cdots \mathrm{C} 14$ & 0.99 & 2.71 & $3.388(4)$ & 126 \\
$\mathrm{C} 72-\mathrm{H} 72 A \cdots \mathrm{Cl} 3$ & 0.99 & 2.78 & $3.617(6)$ & 143 \\
$\mathrm{C} 38-\mathrm{H} 38 \cdots \mathrm{Cl} 1$ & 0.95 & 2.70 & $3.376(4)$ & 129 \\
$\mathrm{C} 33-\mathrm{H} 33 B \cdots C g 3$ & 0.99 & 2.97 & $3.703(5)$ & 132 \\
$\mathrm{C} 69-\mathrm{H} 69 A \cdots C g 8$ & 0.99 & 2.91 & $3.649(6)$ & 132 \\
$\mathrm{C} 60-\mathrm{H} 60 B \cdots C g 3^{\mathrm{i}}$ & 0.98 & 2.84 & $3.655(6)$ & 142 \\
$\mathrm{C} 24-\mathrm{H} 24 B \cdots C g 8^{\mathrm{ii}}$ & 0.98 & 2.85 & $3.710(6)$ & 147
\end{tabular}

Symmetry codes: (i) $x-1, y, z$; (ii) $x+1, y, z$. 